Aus der Abteilung Allgemeinmedizin

(Prof. Dr. med. M.M. Kochen, MPH, FRCGP)

im Zentrum Innere Medizin

der Medizinischen Fakultät der Universität Göttingen

\title{
Bewertung der akademischen Allgemeinmedizin
}

Eine schriftliche Befragung von Hochschulprofessoren

an den deutschen medizinischen Fakultäten

\author{
INAUGURAL - DISSERTATION \\ zur Erlangung des Doktorgrades \\ der Medizinischen Fakultät \\ der Georg-August-Universität zu Göttingen \\ vorgelegt von \\ Tanja Josupeit \\ aus \\ Gießen
}

Göttingen 2002 
D e k a n: Prof. Dr. med. M. Droese

I. Berichterstatter: Priv.-Doz. Dr. disc. pol. W. Himmel

II. Berichterstatter/in: Prof. Dr. med. O. Rienhoff

III. Berichterstatter/in:

Tag der mündlichen Prüfung: 6. Januar 2003 


\section{Inhalt}

1 Einleitung 1

2 Stand der Forschung 3

2.1 Was ist Allgemeinmedizin? 3

2.2 Entwicklung der Allgemeinmedizin als Hochschulfach 4

2.3 Institutionalisierung der Allgemeinmedizin 6

2.4 Allgemeinmedizinische Forschung 8

2.5 Allgemeinmedizinische Lehre 10

2.6 Trends und Reformen 11

2.7 Allgemeinmedizin im Ausland - das Beispiel USA 13

$3 \quad$ Fragestellung und Ziel 16

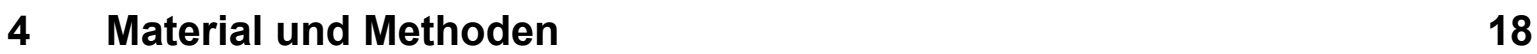

4.1 Methodische Vorüberlegungen 18

4.2 Teilnehmer 19

4.3 Fragebogen 20

4.4 Durchführung der Untersuchung 21

4.5 Datenanalyse 21

5 Ergebnisse $\quad 24$

5.1 Teilnahme 24

5.2 Beschreibung der Stichprobe 24

5.3 Bewertung allgemeinmedizinischer Krankenversorgung, 25 Forschung und Lehre

5.4 Universitäre Institutionalisierung der Allgemeinmedizin 27

5.5 Bewertung der Allgemeinmedizin aus Sicht der Kliniken 29

5.6 Bewertung der Allgemeinmedizin in Abhängigkeit 30 vom Arbeitsort in alten und neuen Bundesländern 
5.7 Bewertung der akademischen Allgemeinmedizin in Abhängigkeit von der persönlichen Bekanntschaft mit dem Vorstand der Abteilung Allgemeinmedizin

5.8 Einflussfaktoren auf die Entscheidung „Stärkung der Allgemeinmedizin im Studium“

6 Diskussion 36

6.1 Methode 36

$\begin{array}{lll}6.2 & \text { Ergebnisse } & 38\end{array}$

6.2.1 Der Allgemeinarzt und chronische Erkrankungen 39

6.2.2 Allgemeinärztliche Entscheidungsfindung 39

6.2.3 Allgemeinmedizinische Forschung 40

6.2.4 Allgemeinmedizinische Lehre 42

6.2.5 Stärkung der Allgemeinmedizin im Studium - Einflussfaktoren 42

6.2.6 Allgemeinmedizin und Innere Medizin 43

6.2.7 Entwicklungen in der Allgemeinmedizin - USA und Europa 45

$\begin{array}{lll}6.3 & \text { Schlussfolgerung } & 46\end{array}$

$\begin{array}{lll}7 & \text { Zusammenfassung } & 47\end{array}$

8 Anhang 48

8.1 Teilnehmende medizinische Fakultäten 48

8.2 Fragebogen 50

8.3 Gesamtauswertung 54

$\begin{array}{lll}8.4 \text { Vollständige Auswertung: } & 60\end{array}$

Bewertung primärmedizinischer Versorgung nach Klinikzugehörigkeit

8.5 Vollständige Auswertung: $\quad 61$

Einstellung zur universitären Allgemeinmedizin nach Klinikzugehörigkeit

9 Literaturverzeichnis 


\section{Einleitung}

Die raschen wissenschaftlichen Fortschritte im Bereich Genetik, Molekularbiologie, Immunologie und sozioökonomische bzw. soziodemographische Veränderungen in den westlichen Industrienationen stellen neue Anforderungen an Ärzte und die medizinische Ausbildung. Künftige Ärzte werden mit einer veränderten Altersstruktur der Patienten und einem daraus resultierenden veränderten Krankheitsspektrum konfrontiert werden. Umweltbelastungen und persönliches Risikoverhalten erfordern eine stärkere Ausrichtung ärztlichen Handelns auf Prävention und Rehabilitation (Wissenschaftsrat 1992).

Vor diesem Hintergrund beschäftigen sich zahlreiche Arbeitsgruppen und Autoren aus dem In- und Ausland mit der Frage nach der Ausbildung des Arztes im 21. Jahrhundert, der den Ansprüchen von Patienten und Gesellschaft gerecht wird. Es besteht Konsens darüber, dass neben der Fähigkeit zu lebenslangem, selbständigem Lernen vor allem primärärztliches Wissen und Können ${ }^{1}$ einen hohen Stellenwert im Medizinstudium haben sollten (Wissenschaftsrat 1992, Murrhardter Kreis 1995, Bundesministerium für Gesundheit 1997, Jolly und Rees 1998, Pauli 1996, Perleth 1998, Bundesärztetag 1996).

Obwohl die Allgemeinmedizin seit 1978 Pflicht- und Prüfungsfach ist (Fischer et al. 1993), gab es 1999 lediglich an sechs der 37 medizinischen Fakultäten einen eigenen Lehrstuhl für Allgemeinmedizin (Kochen 1999). Bis März 2002 ist lediglich ein neuer Lehrstuhl an der Universität Kiel eingerichtet worden. Die Stellungnahme des Wissenschaftsrates vom Januar 1999 zu den Perspektiven des Faches Allgemeinmedizin an den Hochschulen erachtet „eine flächendeckende Etablierung der allgemeinmedizinischen Lehre an allen medizinischen Fakultäten und Hochschulen für erforderlich, um Allgemeinmedizin zu einem verbindlichen Bestandteil der universitären Ausbildung werden zu lassen“ (Wissenschaftsrat 1999, S. 51/52).

Praktische Unterstützung erfährt diese Position durch die im Herbst 1999 vom Bundesministerium für Forschung und Bildung ausgeschriebene Förderung der Forschung in der Allgemeinmedizin. Da eine Gewährleistung der vielfältigen allge-

\footnotetext{
${ }^{1}$ In Deutschland wird die primärärztliche Versorgung hauptsächlich durch Allgemeinärzte und praktische Ärzte, aber auch hausärztlich tätige Internisten und Pädiater gewährleistet (Scheffner 1997).
} 
meinmedizinischen Aufgaben an eine adäquate Etablierung der Allgemeinmedizin an den Hochschulen gebunden ist, werden finanzielle Zuwendungen unter der Voraussetzung gewährt, dass „die Hochschulen bereit sind, einen Beitrag zur Stärkung der Ressourcen und des Leistungsangebotes der Allgemeinmedizin in ihrem universitären Kontext und ihrem Praxisumfeld zu erbringen" (BMBF 1999, S. 2).

Die Einstellung zur Allgemeinmedizin, ihre Akzeptanz und Unterstützung seitens universitärer Entscheidungsträger sind entscheidende Kriterien für die zukünftige Entwicklung und Rolle des Faches an den medizinischen Hochschulen (Grupp 1997, Helmich 1997, Wissenschaftsrat 1992) und Gegenstand dieser Arbeit. 


\section{Stand der Forschung}

\subsection{Was ist Allgemeinmedizin?}

Die exakte Definition des Faches Allgemeinmedizin ist auch bei Fachvertretern immer noch umstritten (Abholz 2000a, Abholz 2000b). Häufig sieht sich die Allgemeinmedizin dem Vorwurf ausgesetzt, nicht mehr zu sein als ein „Sammelsurium" von Inhalten anderer Fachgebiete auf niedrigerem Niveau. Selbst wenn die Allgemeinmedizin immer wieder auf das Wissen und die Techniken anderer Fachgebiete zurückgreift, erlangt dieses Wissen im Kontext allgemeinmedizinischer Aufgaben eine neue Bedeutung, die die Eigenständigkeit der Allgemeinmedizin als Fach rechtfertigt (Perleth 1998).

Die "Deutsche Gesellschaft für Allgemeinmedizin und Familienmedizin“ (DEGAM) definiert: „Allgemeinmedizin ist die Akut- und Langzeitbehandlung von kranken Menschen mit körperlichen und seelischen Gesundheitsstörungen und die ärztliche Betreuung Gesunder unabhängig von Alter und Geschlecht unter besonderer Berücksichtigung der Gesamtpersönlichkeit, der Familie und der sozialen Umwelt" (zitiert nach Klimm 1994, S. 635).

Zur näheren Beschreibung eignet sich das Mehrebenen-Modell, das Hungeling 1995 entworfen hat:

\section{- „Multidisziplinäre“ Ebene}

Allgemeinmedizin erfordert Kenntnisse aus unterschiedlichen Disziplinen der Medizin, die nicht einem additiven Teilwissen, sondern einem sinnvoll vernetzten fächer-übergreifenden Wissen entsprechen. Berücksichtigt werden in gleichem Maße naturwissenschaftliche wie psychosoziale Inhalte, um damit in einem integrierenden Modell dem Wohl des Patienten zu dienen.

- „Bio-psycho-soziale“ Ebene

Aufgabe der Allgemeinmedizin ist die ganzheitliche Patientenbetreuung, in der die biologischen, psychologischen und sozialen Dimensionen von Gesundheit und Krankheit in Hinblick auf den individuellen Patienten gleichermaßen berücksichtigt werden. 
- „Public Health“ -Ebene

Allgemeinärzte sind Schnittstelle und Vermittler zwischen Patient und Gesundheitssystem. Ihnen obliegt die medizinische Grundversorgung der Bevölkerung. Sie haben gesundheitsfördernde und sozialmedizinische Funktionen und erfüllen eine wichtige, koordinierende Aufgabe im öffentlichen Gesundheitswesen.

Die Allgemeinmedizin ergänzt das in der klinischen Medizin vorherrschende biologische Modell, das die Krankheit des Patienten in den Mittelpunkt stellt, um einen integrativen „bio-psycho-sozialen“ Ansatz (Engel 1982), das den Patienten in seiner ganzen Persönlichkeit und seinem Umfeld betrachtet. Allgemeinmedizin ist daher kein „Sammelsurium“ von verschiedensten medizinischen Inhalten, sondern eine „integrative Sicht- und Denkweise“ (Goßmann 1992, Comberg 1994, Klimm 1994).

Im Mittelpunkt der Allgemeinmedizin steht das mehrdimensionale Patientenproblem, wobei die eigentliche Krankheit gegenüber psychischen oder sozialen Anliegen in den Hintergrund treten kann (Himmel et al. 2002). Die Patienten in einer Allgemeinarztpraxis sind unausgelesen; und die technische Ausstattung der Praxis steht zumeist nicht im Vordergrund (Murrhardter Kreis 1995), obwohl viele Praxen durch Zusatzbezeichnungen und zusätzliche diagnostische Möglichkeiten keineswegs das Bild von „Barfußmedizin“ aufkommen lassen (Bödecker 2000). Oft kann der Hausarzt durch seine langjährige Kenntnis des Patienten einen individuellen Behandlungsansatz anbieten (Abholz 1998).

\subsection{Entwicklung der Allgemeinmedizin als Hochschulfach}

Die Allgemeinmedizin ist als Stoffgebiet im Rahmen des Medizinstudiums relativ neu. Zu Beginn der 60er Jahre des 20. Jahrhunderts fanden erstmals Vorlesungen in Allgemeinmedizin statt; 1966 wurde in Freiburg der erste Lehrauftrag vergeben (Fischer et al. 1993). 1978 wurde die Allgemeinmedizin durch Änderung der Approbationsordnung zum Pflicht- und Prüfungsfach für die schriftlichen Examina, seit 1988 ist sie auch mündliches Prüfungsfach im 2. und 3. Staatsexamen (Wissenschaftsrat 1999). 
Allgemeinmedizin wird als Pflichtkurs (an der Universität Göttingen z.B. 26-stündig) in den höheren klinischen Semestern gelehrt. Darüber hinaus bieten viele Universitäten über die Abteilungen für Allgemeinmedizin weitere fakultative Unterrichtsangebote wie z.B. Seminare zu Gesundheitswissenschaften oder Praktika für Famulaturen in der Allgemeinarztpraxis an (Vereinigung der Hochschullehrer... 2000/2001). Die meisten Abteilungen für Allgemeinmedizin sind außerdem an der Gestaltung der obligaten vorklinischen Veranstaltungen "Einführung in die Klinische Medizin“ und „Berufsfelderkundung“ beteiligt (Kochen et al. 1990). Die Entwürfe zur neuen Approbationsordnung sehen neben der Einführung eines Blockpraktikums in Allgemeinmedizin die Möglichkeit vor, Allgemeinmedizin als Wahlfach im Praktischen Jahr zu absolvieren; beides wird ab dem Wintersemester 2003/2004 Bestandteil des reformierten Medizinstudiums sein (Bundesministerium für Gesundheit 1997, Bundesministerium für Gesundheit 2001, Bundesministerium für Gesundheit 2002).

Die Integration der Allgemeinmedizin in den universitären Alltag wird seit den Anfängen von politischen und standespolitischen Diskussionen begleitet. Bereits 1969 forderte die Kultusministerkonferenz der Länder die Einführung der Allgemeinmedizin in den Ausbildungskatalog und die Schaffung der entsprechenden Lehreinrichtungen (Fischer et al. 1993). Im September 1989 fasste die Kultusministerkonferenz den Beschluss, bis Anfang der 90er Jahre an allen deutschen medizinischen Hochschulen ordentliche Lehrstühle für Allgemeinmedizin einzurichten (Carter und Elvyn 2002, Kochen et al. 1990). Die Gesundheitsministerkonferenz stellte im Mai 1995 fest: „Jede medizinische Fakultät sollte einen Lehrstuhl für Allgemeinmedizin haben" (zitiert nach Wissenschaftsrat 1999, S. 3). Auch der Deutsche Ärztetag forderte in den letzten Jahren wiederholt eine stärkere Intergration allgemeinmedizinischer Inhalte in die medizinische Ausbildung und die flächendeckende Einrichtung von Lehrstühlen für Allgemeinmedizin (Bundesärztetag 1996).

Trotz dieser langjährigen Forderungen ist die Integration der Allgemeinmedizin in den universitären Alltag nur bedingt gelungen und die Institutionalisierung ist auch 30 Jahre nach Erteilung des ersten Lehrauftrages noch nicht an allen Universitäten umgesetzt. 


\subsection{Institutionalisierung der Allgemeinmedizin}

Im Juli 1997 hatten fünf von 36 deutschen medizinischen Fakultäten eine volle C4Professur für Allgemeinmedizin: FU Berlin, Düsseldorf, Frankfurt, Göttingen und Hannover (Wissenschaftsrat 1999). Bis März 2002 kam lediglich eine weitere C4Professur an der Christian-Albrechts-Universität Kiel hinzu.

An den anderen Universitäten wird die Lehr- und Prüfungstätigkeit meist durch Lehrbeauftragte sichergestellt, deren Anzahl zwischen einem und 20 Lehrbeauftragten je nach Universität schwanken kann. Es existieren auch „Mittelwege“, die die Allgemeinmedizin in Form von halben C4-Professuren (Ulm), C3-Professuren (Heidelberg, Leipzig, Marburg, Berlin Charité) oder Honorar- bzw. apl. Professuren (Aachen, Bonn, Essen) universitär etabliert haben (Vereinigung der Hochschullehrer... Wintersemester 2000/2001).

Die Vertretung der Allgemeinmedizin allein durch Lehrbeauftragte wird für ein akademisches Fach mit Lehr- und Prüfungstätigkeit und einem Forschungsauftrag von den meisten Kommentatoren als ungenügend bewertet. Ein auf Zeit vergebener Lehrauftrag mit meist unzureichender Vergütung kann keine ausreichende Absicherung der vielfältigen inhaltlichen und organisatorischen Aufgaben gewährleisten (Murrhardter Kreis 1995). In Zukunft werden die geplanten Reformen der Approbationsordnung mit der Einführung von Blockpraktika in Allgemeinmedizin (in Göttingen seit 2000; Abteilung Allgemeinmedizin Göttingen 2002) und eine Beteiligung am Praktischen Jahr eine große Herausforderung darstellen, die ohne etablierte und personell wie finanziell gut ausgestattete Abteilungen schwer zu bewältigen sein wird (Gulich und Zeitler 1999).

Auch für die Forschungstätigkeit in der Allgemeinmedizin ist die befriedigende universitäre Institutionalisierung Voraussetzung. Berichte aus dem Ausland stellen die wichtige koordinierende Funktion der universitären Allgemeinmedizin für die Forschung im Rahmen von ambulanten Studien heraus (Morrison und Watt 2001). Eine deutsche Studie zum Habilitationswunsch bei Allgemeinärzten ermittelte als entscheidende Erwartung an die universitäre Allgemeinmedizin Unterstützung und Beratung in Forschungsfragen (Himmel und Kochen 1997). 
In der ehemaligen DDR war die Allgemeinmedizin an allen Fakultäten in Form von ambulanten Polikliniken verankert, die gleichberechtigt neben den niedergelassenen Ärzten an der Krankenversorgung teilnahmen (Murrhardter Kreis 1995). Ein Hauptanliegen des sogenannten sozialistischen Gesundheitssystems war schon seit den 50er Jahren die allgemeine und umfassende medizinische Grundbetreuung der Bevölkerung und somit auch eine Förderung des Allgemeinarztes als Garanten für eine kontinuierliche ambulante Versorgung (Günther 1990, Maronde und Sander 1989). Bereits 1967 wurde der Facharzt für Allgemeinmedizin geschaffen und damit den anderen Fachärzten gleichgestellt (Harych 1990, Niemann und Wiezorek 1980a, Niemann und Wiezorek 1980b, Pönicke 1990). Seit Mitte der 60er Jahre gab es ein obligates „Berufspraktikum Allgemeinmedizin“, in das Vorlesungen von Allgemeinärzten integriert waren (Bühler 1998). Auch die Frage, wie mehr Studenten für eine allgemeinärztliche Laufbahn zu gewinnen seien, ist in den 80er Jahren diskutiert und durch Befragungen von Studenten konkretisiert worden (Maronde und Sander 1989). Allerdings wurde die Allgemeinmedizin erst 1989 als offizielles universitäres Lehrfach im Curriculum genannt (Bühler 1998).

Die schleppende Institutionalisierung wird u.a. auf einen Mangel an „universitären Gütekriterien“ zurückgeführt. Es fehlen Forschung und spezifische Wissenschaft, die durch Promotion und Habilitation den akademischen Nachwuchs heranbilden (Ferber 1989). Das Fehlen einer akademischen Allgemeinmedizin birgt nicht nur Konfliktpotential für das Bestehen in der universitären Umgebung (Habeck 1992), sondern führt auch zu Unsicherheiten in der Diskussion um die adäquate Qualifikation eines Lehrstuhlinhabers für Allgemeinmedizin (Kochen 2002). Im Moment mangelt es an Bewerbern, die die klassische akademische Laufbahn mit Habilitation absolviert haben. In der Debatte um die Anforderungen an einen zukünftigen Professor für Allgemeinmedizin (Kochen 1989) stehen auf der einen Seite die Forderungen nach Habilitation und Erfahrungen in einer eigenen Praxis (Ferber 1989), auf der anderen Seite herrscht die Ansicht, langjähriger Lehrauftrag und Leitung einer eigenen Kassenarztpraxis seien ausreichende Eignung für einen solchen Posten (Hagedorn 1989). Diese Unentschiedenheit spiegelt sich auch im kontrovers diskutierten Weg für die Ausbildung des wissenschaftlichen Nachwuchses wider (Hummers-Pradier et al. 2002). 
Auch der Bericht des Wissenschaftsrates von 1999 führt die akademischen Anforderungen an einen Abteilungsdirektor nicht exakt aus. Gefordert werden lediglich "Berufserfahrung in der allgemeinmedizinischen Praxis und Lehre" sowie die Aufrechterhaltung einer (eingeschränkten) praktischen Tätigkeit nach Übertragung des Professorenamtes. Der klassische Weg der Habilitation, wie er in anderen Fächern und auch im deutschsprachigen Ausland die Regel ist (bzw. war, wenn man von einer schnellen Durchsetzung des Modells „Juniorprofessur“ ausgeht), wird nicht als Auswahlkriterium angeführt.

In einer Befragung der Mitglieder der DEGAM, der wissenschaftlichen Gesellschaft für Allgemein- und Familienmedizin, zum Thema „Habilitationswunsch“ äußerte eine Reihe von Allgemeinärzten, hier vor allem jüngere Ärzte, Interesse an einer Habilitation (Himmel und Kochen 1997). Ob die Habilitation allerdings der beste Weg für die wissenschaftliche Allgemeinmedizin ist, ist nicht unumstritten. Denn Länder mit einer starken und wissenschaftlich sehr aktiven Allgemeinmedizin wie z.B. Großbritannien und die Niederlande haben ein weniger reglementiertes System postgradualer wissenschaftlicher Ausbildung (in allen Fächern) und sind damit sehr erfolgreich (Kochen und Himmel 2000).

\subsection{Allgemeinmedizinische Forschung}

Die Allgemeinmedizin versteht sich als ganzheitlich betreuendes, patientenorientiertes Fach. Anders als die biomedizinische Forschung der klinisch-experimentellen Fächer basiert ihre Forschung nicht nur auf "harten“, objektivierbaren Daten, die unter Laborbedingungen erhoben wurden (Kochen 1998).

Allgemeinmedizinische Forschung bedient sich in vielen Fällen der Methoden der empirischen Sozialforschung, ähnlich wie auch Sozialmedizin, Medizinische Soziologie und Medizinische Psychologie. Eine weitere Gemeinsamkeit dieser Fächer ist ihre vergleichsweise marginale Rolle im medizinischen Curriculum. Gesundheitswissenschaften, ein neues Fach, das mit der Allgemeinmedizin zentrale Ziele und Methoden teilt, ist nicht Bestandteil der medizinischen Ausbildung. Die Möglichkeit für Studenten und Hochschullehrer mit sozialwissenschaftlichen Forschungsthemen und -methoden in Kontakt zu kommen, ist daher nur in begrenztem Rahmen gegeben (Abholz 1997). 
Neben den Methoden unterscheidet sich allgemeinmedizinische Forschung auch von der klinischen Forschung durch den Ort, an dem sie stattfindet: Statt Klinik oder Labor ist die ärztliche Praxis im Rahmen der ambulanten Versorgung Ort der Forschung. Die Forschungsideen entwickeln sich in diesem Umfeld und aus typischen Behandlungsanlässen, die in der Klinik oftmals nicht existieren. Allgemeinmedizinische Forschung kann sehr wohl klinische Forschung sein, d.h., klinische Fragestellungen bearbeiten. Die Besonderheiten der Praxis (z.B. epidemiologische Verteilung) prägen ihre Ergebnisse. Sie kann daher nicht ohne weiteres durch klinische Forschung im Krankenhaus ersetzt werden. Eine Erforschung allgemeinmedizinischer Themen durch fachfremde klinische Arbeitsgruppen schränkt die Übertragbarkeit der Ergebnisse ebenfalls ein, da die Besonderheiten der hausärztlichen Medizin nicht ihrer Bedeutung entsprechend berücksichtigt werden können (Kochen et al. 2000).

Die Forschungsthemen der Allgemeinmedizin gliedern sich nach Kochen (1998) in fünf große Bereiche:

- Epidemiologische Forschung

Krankheitsentstehung, -verteilung und -entwicklung in der Bevölkerung

- Gesundheitsversorgungsforschung:

Analyse medizinischer und psychosozialer Versorgung in der allgemeinärztlichen Praxis und Entwicklung von Optimierungsstrategien

- Arzt-Patient-Beziehung/Familienforschung:

Untersuchung sozialer Interaktion und Verhaltensweisen in der allgemeinärztlichen Praxis und ihre Bedeutung für die allgemeinärztliche Tätigkeit

- Bildungs- und Qualifikationsforschung:

Evaluation der Aus-, Weiter- und Fortbildung und Lehre in der Allgemeinmedizin

- Medizinische Forschung in der Allgemeinmedizin:

Übertragung klinischer Untersuchungsmethoden und Studien auf den ambulanten Bereich um die Relevanz klinischer Ergebnisse für die Praxistätigkeit zu sichern.

Trotz eines quantitativen Aufschwungs bis in die Mitte der 80er Jahre zeichnet eine Bestandsaufnahme ein unvorteilhaftes Bild von der Qualität allgemeinmedizinscher Forschung in Deutschland: Vielen Studien mangele es an Wissenschaftlichkeit, 
Repräsentativität und Vergleichbarkeit (Brüning 1987). Bis Mitte der 90er Jahre registriert Joosten (1995) einen Rückgang allgemeinmedizinischer Publikationen. Zu diesem Zeitpunkt wurde lediglich an neun medizinischen Hochschulen allgemeinmedizinische Forschung betrieben, hier vor allem an den Universitäten mit eigenem Lehrstuhl (Fischer et al. 1993).

Ein Vergleich allgemeinmedizinischer Zeitschriften, vor allem aus dem angloamerikanischen Sprachraum signalisiert Nachholbedarf. Der allgemeine Trend zeigt jedoch heute einen qualitativen wie quantitativen Aufschwung in der allgemeinmedizinischen Forschung und ein breites Interesse auch anderer Disziplinen an deren Ergebnissen (Kochen et al. 2000).

\subsection{Allgemeinmedizinische Lehre}

Obwohl an Universitätskliniken, also Häusern der Maximalversorgung, lediglich 1\% aller tatsächlichen Arzt-Patient-Kontakte stattfinden - $99 \%$ aller Kontakte erfolgen ambulant -, ist das Medizinstudium überwiegend in diesem durch hochselektierte Krankheitsbilder, Apparatemedizin und kurze Patientenkontakte geprägten Umfeld angesiedelt („Eisbergphänomen“; Last 1963, Braun 1988, van de Lisdonk 1989, Kochen et al. 1990). Gegenstand der Wissensvermittlung sind in der klassischen klinischen Ausbildung die klar definierte Krankheit und der Patient als Träger dieser Krankheit (Wissenschaftsrat 1999).

Die Ausbildungsziele der Allgemeinmedizin orientieren sich am individuellen Patienten, den Bedürfnissen der Bevölkerung und am Berufsfeld des Arztes in der Primärversorgung. Im Vordergrund steht das Erlernen ärztlichen Handelns (Wissenschaftsrat 1999). Der Anteil ambulanter Versorgung an der medizinischen Ausbildung beschränkt sich derzeit noch auf eine Pflichtfamulatur von einem Monat bei einem beliebigen niedergelassenen Arzt. Ein Kontakt mit der praktischen Allgemeinmedizin konnte lange Zeit im Medizinstudium völlig fehlen (Bundesärztekammer 1994). Im Lehrstoffkatalog Allgemeinmedizin ist den Besonderheiten der Allgemeinmedizin in der Formulierung von Lernzielen für den Rahmen des Unterrichts an den Universitäten Rechnung getragen worden. Wichtige Lehr- und Lerninhalte betreffen die Position von Allgemeinärzten im Gesundheitssystem, so die primärärztliche Sieb- und Notfallfunktion, die Koordinationsfunktion, die haus- und 
familienärztliche Betreuung, die Gesundheitsbildungsfunktion, die soziale Integrationsfunktion sowie ökologische und ökonomische Funktionen (Habeck 1992).

Das zentrale Anliegen allgemeinmedizinischer Lehre ist nicht allein das Vermitteln von Fakten. Ziel ist es, durch Erkennen von Zusammenhängen und Herausarbeiten von Handlungsstrategien eine Verhaltensänderung bei den Studenten zu erreichen (Härter et al. 1990). Das Vorleben praktischen medizinischen Handelns und die Erfahrung eines spezifisch-allgemeinmedizinischen Denkstils weisen dem einzelnen lehrenden Arzt hierbei eine entscheidende Rolle als Vorbild für seine Studenten zu (Hungeling 1995, Schrömbgens 1990, Sahlmann 2000).

Die Formung einer „Arztpersönlichkeit“ durch „Situationen, die Gelegenheit zur Selbsterfahrung, Selbstveränderung und zum gemeinsamen Diskurs über Maßstäbe ärztlichen Handelns geben", ist in der medizinischen Lehre (medical education) gefordert und erwünscht (Murrhardter Kreis 1995, S. 127), und könnte gerade im allgemeinmedizinischen Curriculum gefördert werden.

\subsection{Trends und Reformen}

Die Fortschritte in der Grundlagenforschung der Medizin der letzten Jahre führ(t)en zur immer weiterreichenden Spezialisierung der Ärzteschaft. Noch 1991 entsprach das Verhältnis Haus- zu Facharzt bei den niedergelassenen Vertragsärzten, dem von der Politik geforderten Verhältnis von 60 zu 40 Prozent (Kopetsch 2002). Im Jahr 2000 hingegen lag der Anteil von Spezialisten bei 48\% (Kassenärztliche Bundesvereinigung 2001). In Hinblick auf die Veränderungen der Bevölkerungsstruktur, des Krankheitsspektrums und nicht zuletzt der finanziellen Ressourcen fürchtet man in Zukunft eine unzureichende ärztliche Versorgung, besonders im hausärztlichen Bereich. Dort werden altersbedingt in den nächsten Jahren über 20.000 Ärzte aus der Krankenversorgung ausscheiden. In den neuen Bundesländern ist dieser Trend jetzt schon zu bemerken. Der Bedarf an Allgemeinärzten, die auch zukünftig die primärmedizinische Grundversorgung sicherstellen, ist groß. Allerdings nimmt die Anzahl nachrückender, junger Ärzte kontinuierlich ab (Kopetsch 2002). Wie auch in den USA (Kapitel 2.7) wird die Gesundheitspolitik Anreize schaffen müssen, um junge Ärzte für die Allgemeinmedizin zu begeistern. Studien haben gezeigt, dass vor allem starke universitäre Abteilungen und langjährige Kontakte mit der Allgemein- 
medizin, das Interesse in diesem Bereich zu arbeiten, signifikant beeinflussen (Wilms 1997).

Für Deutschland ergibt sich nicht nur das Problem der mangelhaften Institutionalisierung der Allgemeinmedizin an den Hochschulen. Problematisch ist auch die Weiterbildung: Während in den angloamerikanischen Ländern ein strukturiertes Weiterbildungscurriculum für Allgemeinmedizin in festen Klinik- und Praxisverbünden existiert, ist der deutsche Arzt in allgemeinmedizinischer Weiterbildung auf sich selbst gestellt (Donner-Banzhoff und Abholz 2000). Im medizinischen Miteinander wird der Beschluss des Deutschen Ärztetages von 1997, die Weiterbildungszeit in der Allgemeinmedizin von drei auf fünf Jahre zu verlängern, zu einer Gleichstellung mit anderen Fachgebieten beitragen. Allerdings werden die zusätzlichen Anforderungen die Situation eher verschärfen. Beispielsweise ist eine pädiatrische Weiterbildungsstelle schon für Assistenten, die in der Pädiatrie bleiben wollen, schwierig zu finden (DEGAM 2002a, DEGAM 2002b). Der Entschluss der Gesundheitsministerkonferenz vom Mai 1998 zur Sicherstellung der allgemeinmedizinischen Versorgung soll die Umsetzung der neuen Weiterbildungsordnung erleichtern (Kochen 1999). Dieses Initiativprogramm läuft 2003 aus. Die Daten lassen allerdings erwarten, dass ein großer Bedarf auch für die Zeit nach 2003 bestehen wird, sodass Veränderungen und Anreize auch über die Laufzeit dieses Programmes hinaus erforderlich sind (Kopetsch 2002).

Überlegungen zur effektiveren Gestaltung des Gesundheitswesens führen immer wieder zum Thema „Primärarztsystem“ (Starfield 1991, Abholz 1999, Himmel et al. 2000, Engström et al. 2001). Länder, die auf einer breiten primärärztlichen Basis agieren, haben niedrigere Ausgaben im Gesundheitswesen, ihre Bevölkerung ist zufriedener mit ihren Ärzten, braucht weniger Medikamente und ist im Schnitt gesünder (Starfield 1994, Korzilius 2001a, Korzilius 2001b). Basis eines solchen Systems ist der gut ausgebildete Allgemein- oder Hausarzt als zentrale Kontaktperson des Patienten im Gesundheitswesen. Voraussetzung hierfür ist ein verändertes Aus- und Weiterbildungssystem mit hohen Qualitätstandards und einer deutlichen Stärkung allgemeinmedizinischer Inhalte (Marzi und Abholz 1999). Bezeichnenderweise ist in Ländern mit etabliertem Primärarztsystem wie z.B. den Niederlanden oder Großbritannien die Allgemeinmedizin ein an der Universität fest verankertes Fach, das die Aus- und Weiterbildung koordiniert (Steinkohl1996). 
Reformbestrebungen zum Medizinstudium fordern vor dem Hintergrund dieser Veränderungen eine Stärkung allgemeinmedizinischer und primärärztlicher Inhalte auch in Deutschland (Clade 1997a, Clade 1997b, Murrhardter Kreis 1995, Wissenschaftsrat 1992). Die seit 1997 geplante und 2002 zu verabschiedende Novelle der ärztlichen Approbationsordnung soll diesen Anforderungen Rechnung tragen (Clade 2001, Richter 2001a, Bundesministerium für Gesundheit 2002). Der Arzt von morgen braucht ärztliche Basiskompetenzen, die angesichts einer Flut von Wissen und administrativer Anforderungen, den Anspruch patientenorientierter Medizin einlösen. Zu diesen Basiskompetenzen zählen nach Perleth (1998):

- Psychosoziale und kommunikative Kompetenzen/Beratung und Information

- Primärmedizin

- Sozialmedizin

- Medizinethik

- Klinische Epidemiologie.

Grundlegend für den Arzt des 21. Jahrhunderts ist eine neue Konzeption zum Verständnis von Medizin: „Gesundheit und Krankheit müssen [...] als ein komplexes, vielfach verwobenes Gefüge verstanden werden, in dem biologische, psychologische und soziale Elemente von Gesundheit und Krankheit als gleichwertige Bedingungen der menschlichen Existenz zu begreifen sind“ (Wissenschaftsrat 1992, S. 43).

\subsection{Allgemeinmedizin im Ausland - das Beispiel USA}

Die Zahl der Studenten mit Berufsziel Allgemeinarzt ist in Deutschland rückläufig (Wilm 1997). Dieser Trend, der auch im europäischen und amerikanischen Ausland bestand, wurde erstmals 1993 in den USA durchbrochen (Sullivan und Morrison 1997) - nicht zuletzt als Ergebnis von Untersuchungen über Probleme des amerikanischen Gesundheitssystems: Regierung, Gesundheitsindustrie und akademische Medizin sahen übereinstimmend einen gesteigerten Bedarf der Bevölkerung an Allgemeinärzten (Curtis und Smith 1997). Aus dieser Erkenntnis resultierten Initiativen zur Steigerung der Zahl der Allgemeinärzte. Die meisten Ansätze basierten auf der Annahme, dass die medizinische Ausbildung der Schlüssel sei, um primärärztliche Versorgung an Attraktivität gewinnen zu lassen.

Zahlreiche Studien belegen, dass neben persönlichen Eigenschaften von Studenten vor allem zwei Faktoren einen großen Einfluss auf eine spätere allgemein- 
medizinische Orientierung haben (Bland et al. 1995, Senf et al. 1997, Sullivan und Morrison 1997, Wilm 1997, Howe und Galen 2001): das Vorhandensein einer starken allgemeinmedizinischen Abteilung an der Hochschule und praktische Erfahrungen in der Allgemeinmedizin über einen längeren Zeitraum.

Um den Bedarf an Allgemeinärzten zu decken, wurde in den USA 1991 von der „Robert Wood Johnson Foundation“ die „Generalist Physician Initiative“ ins Leben gerufen. 1999 wurden die ersten Ergebnisse verschiedener Hochschulen, die an diesem Projekt teilnahmen, veröffentlicht. Allen Universitäten war es gelungen, die Zahl der Studenten, die nach ihrem Abschluss eine Weiterbildung in Allgemeinmedizin wählten, zu steigern. Allerdings stieg in den 90er Jahren insgesamt das Interesse an allgemeinmedizinischer Weiterbildung, sodass das positive Ergebnis möglicherweise nicht allein auf die „Generalist Physician Inititiative“ zurückzuführen ist (Blake 1999).

Trotz der Erfolge der Programmteilnehmer (Grayson et al. 1999, Brooks et al. 1999) und der Tatsache, dass es in den USA schon sehr viel länger als in Deutschland eine etablierte und aktive Allgemeinmedizin an den Hochschulen gibt, hat das Fach auch in den Vereinigten Staaten mit Vorurteilen zu kämpfen. Problemfelder im interdisziplinären Miteinander sind nach Curtis und Smith (1997):

1. Unsicherheit über die Definition und Rolle des Allgemeinarztes

2. Schlechter klinischer Ruf der im Krankenhaus tätigen Allgemeinärzte

3. Fehlende wissenschaftliche Grundlagen

4. Unklare Finanzierung der Projekte zur Verbesserung der allgemeinmedizinischen Ausbildung.

Eine Studie über die Einstellungen („attitudes“) von akademischen Lehrern und Studenten zur Primärversorgung an amerikanischen Hochschulen sah ein eher „frostiges Klima“ für die Weiterentwicklung der Primärversorgung an den Universitäten (Block et al. 1996). Die Befragten äußerten überwiegend negative Haltungen zur Primärmedizin: allgemeinärztliche Tätigkeit erfordere lediglich eine geringe Qualifikation, die Qualität der Forschung sei schlechter als in anderen Fächern, Studenten mit dem Berufswunsch Allgemeinarzt würden darin kaum ermutigt. 
Eine follow-up Untersuchung bei Studenten und Weiterbildungsassistenten, die an der oben zitierten Studie teilgenommen haben, deutet eine positive Entwicklung an (Zinn et al. 2001). Zwar wurde im Laufe von Studium und Weiterbildung die primärmedizinische Orientierung der Befragten schwächer; andererseits empfanden sie die Haltung der Lehrenden zur Primärmedizin positiver als in der Ausgangsbefragung von 1994. Dies könnte ein Hinweis darauf sein, dass trotz positiver Verstärkung durch die medizinischen Lehrer im Studium, die klinischen Alltagserfahrungen den Berufswunsch Allgemeinarzt „dämpfen“.

Auch medizinische Hochschulen, die traditionsgemäß primärmedizinisch ausgerichtet sind und Studenten zu einer Laufbahn in der Allgemeinmedizin ermutigen, können eher kritische Einstellungen ihrer Studenten zur Allgemeinmedizin nicht verhindern (Block et al. 1998).

Ob die zunächst erfolgreiche Förderung der Ausbildung von Allgemeinärzten durch organisierte Programme einen Einfluss auf die Haltung zur Primärmedizin haben wird, bleibt - in Deutschland wie in den USA - abzuwarten. Die Vergabe der Weiterbildungsplätze in den USA der Jahre 2000/2001 verweisen auf eine eher negative Entwicklung: In den letzten drei Jahren hat die Zahl junger Ärzte, die sich für eine Weiterbildung in Allgemeinmedizin entschieden haben, kontinuierlich abgenommen (Pugno et al. 2000, Pugno et al. 2001). 


\section{$3 \quad$ Fragestellung und Ziel}

Obwohl die Allgemeinmedizin in Deutschland seit 20 Jahren fest im medizinischen Curriculum verankert ist, ist sie an den deutschen medizinischen Fakultäten immer noch lückenhaft etabliert. Im akademischen Alltag begegnet sie Vorurteilen und muss im interdisziplinären Miteinander Eigenständigkeit und Existenzberechtigung deutlich betonen.

Initiativen zur Gestaltung eines modernen Medizinstudiums und gesundheitspolitische Überlegungen, die vor dem Hintergrund der stetig steigenden Kosten im deutschen Gesundheitswesen ein „primary care led“-System favorisieren (WHO 1978, Coulter 1996, Starfield 1997), betrachten allgemeinmedizinische Inhalte und Kenntnisse als unverzichtbare Bestandteile der medizinischen Aus- und Weiterbildung.

Auf die Forderungen zur Stärkung der Allgemeinmedizin ist in den letzten zwei Jahren mit Förderprogrammen sowohl im universitären als auch im ambulanten Bereich reagiert worden. Allerdings ist die Inanspruchnahme und Umsetzung dieser Initiativen an den Universitäten z.T. vom Interesse und der Zustimmung der fakultären Entscheidungsträger abhängig.

In seiner Stellungnahme von 1999 veröffentlichte der Wissenschaftsrat eine Kurzumfrage, in der die Fakultäten die Situation der Allgemeinmedizin an ihrer eigenen Fakultät und in Deutschland anhand von einer Ratingskala („sehr gut“ bis „ausreichend“) beurteilen sollten. Von den 1997 angeschriebenen 37 medizinischen Hochschulen haben 35 geantwortet. 39,3\% bewerteten die Situation der Allgemeinmedizin an ihrer Hochschule mit „ausreichend“, 32,1 \% mit „ungenügend“, 10,7\% mit "gut“ und 21,4\% mit „sehr gut“. Zwei Drittel der antwortenden Fakultäten benoteten die deutschlandweite Lage der Allgemeinmedizin mit „ungenügend“, ein Viertel war in der Wertung unentschieden (Wissenschaftsrat 1999). Weiterreichende Informationen bieten diese Daten jedoch nicht.

Im Gegensatz zu den Vereinigten Staaten, wo die Allgemeinmedizin in mehreren Studien durch facheigene und fachfremde Lehrende, medizinische Assistenten und Studenten bewertet wurde, hat es in Deutschland bislang keine Erhebungen über die 
Einstellung von Hochschullehrern zur Allgemeinmedizin an den Universitäten gegeben.

Gegenstand der vorliegenden Untersuchung ist die Bewertung der Allgemeinmedizin durch medizinische Hochschullehrer, deren klinische Abteilungen durch fachliche Nähe, Teilnahme an der Primärversorgung oder psycho-soziale Ausrichtung in einem besonderen Verhältnis zur Allgemeinmedizin stehen. Die Einschätzung der Bedeutung und Qualität allgemeinmedizinischer Forschung und Lehre und die persönlichen Erfahrungen der teilnehmenden Professoren mit der Allgemeinmedizin sollten erfragt werden und ggf. Gruppenunterschiede herausgearbeitet werden.

Neben der Klinikzugehörigkeit als möglichem Einflussfaktor auf das Antwortverhalten liegt ein besonderes Augenmerk auf der persönlichen Bekanntschaft mit dem jeweiligen Leiter der Abteilung für Allgemeinmedizin und dem Arbeitsort der Befragten (neue vs. alte Bundesländer). Die persönliche Bekanntschaft mit dem Abteilungsleiter (bzw. Leiter des Lehrauftrages) wird als Indikator für eine universitär aktive Abteilung für Allgemeinmedizin gesehen. In vielen Studien zum Berufsziel Allgemeinarzt gilt dieser Sachverhalt als positiver Einflussfaktor auf die spätere Berufswahl von Studenten. Eine starke und damit hochschulöffentlich bekannte Abteilung für Allgemeinmedizin dürfte auch im akademischen Umfeld die Beurteilung der Allgemeinmedizin positiv beeinflussen.

In den neuen Bundesländern war die Allgemeinmedizin sowohl in den Universitäten als auch im ambulanten Rahmen stärker vertreten, als dies in den alten Ländern der Fall war und ist. Diese Aspekte können die Fremdwahrnehmung der Allgemeinmedizin verändern und Einfluss auf die Bewertung der akademischen Allgemeinmedizin haben.

Ziel dieser Arbeit ist es, durch ein wissenschaftlich fundiertes Stimmungsbild an den medizinischen Fakultäten Deutschlands die Güte der Allgemeinmedizin in der Fremdwahrnehmung und ihr Entwicklungspotential einzuschätzen. Hieraus könnten sich mögliche Ansätze für eine Förderung der Allgemeinmedizin ergeben. 


\section{$4 \quad$ Material und Methoden}

\subsection{Methodische Vorüberlegungen}

Thema dieser Arbeit ist die Einstellung von medizinischen Hochschullehrern zur Allgemeinmedizin als universitärem Fach. Die Operationalisierung des Begriffes "Einstellung" erfolgte in Anlehnung an die amerikanische Studie von Block et al. (1996), die Einstellungen zur Allgemeinmedizin durch einen für diesen Zweck entworfenen Fragebogen erfragt haben. Um den Besonderheiten der Allgemeinmedizin an deutschen Universitäten gerecht zu werden, wurden Aspekte, die nach Durchsicht der deutschen Literatur (s. Kapitel 2) wichtig erschienen, zusätzlich in die Untersuchung aufgenommen (Bortz 1984).

Untersuchungsinstrument der vorliegenden Arbeit ist die schriftliche Befragung mit einem standardisierten Fragebogen. Diese Methode ist besonders bei großem Umfang und breiter regionaler Streuung der Stichprobe geeignet. Die Homogenität der Gruppe der Adressaten unterstützte als positives Kriterium die Auswahl des Instruments (Friedrichs 1990). Die unkontrollierbare Erhebungssituation und eine möglicherweise geringe Rücklaufquote müssen als Nachteil schriftlicher Erhebungen in Kauf genommen werden (Schnell et al. 1999).

Um die Rücklaufquote zu maximieren, erfolgten inhaltliche und formale Gestaltung von Fragebogen und die persönlichen Anschreiben an die Adressaten nach den Grundsätzen der „total design method“ (TDM), die 1978 von Dillman entwickelt wurde (Dillman 1978, Geyer und Siegrist 1998). Durch diese Optimierung des Instruments und seiner Anwendung können die Defizite der schriftlichen Befragung ausgeglichen und aussagekräftige Daten gewonnen werden (Hippler 1988, Thoma und Zimmermann 1996).

Die Durchführung der Erhebung orientierte sich ebenfalls an den Richtlinien der TDM. Blieb die erste Zusendung des Fragebogens unbeantwortet, erfolgten ein bzw. zwei weitere Aufforderungen: eine telefonische Nachfrage nach drei Wochen und eine schriftliche Erinnerung in Form eines erneuten persönlichen Anschreibens mit Fragebogen nach drei weiteren Wochen. 
Zur Rücklaufkontrolle hatte der Fragebogen ein Deckblatt mit Identifikationsnummer, das zur Wahrung der Anonymität der Teilnehmer sofort nach Eingang des Fragebogens entfernt wurde. Die Dateneingabe erfolgte ohne diese Identifikationsnummer.

\subsection{Teilnehmer}

Adressaten dieser Studie waren die Dekane und Direktoren ausgewählter klinischer Abteilungen an den 37 deutschen medizinischen Fakultäten in ihrer Funktion als Hochschullehrer, Forscher und innerfakultäre Entscheidungsträger. Die Direktoren waren Leiter der Abteilungen für Chirurgie, Innere Medizin, Pädiatrie, Gynäkologie und Psychiatrie/Psychosomatik.

Die Auswahl der klinischen Fächer wurde durch eine besondere Beziehung des Faches zur Allgemeinmedizin bestimmt: Innere Medizin, Chirurgie und Pädiatrie sind die wichtigsten Fächer in der Weiterbildung zum Allgemeinarzt (Musterweiterbildungsordnung, Bundesärztetag 1997). Gynäkologie und Psychiatrie/Psychosomatik sind fakultative Fächer der Weiterbildung. Die ambulante Gynäkologie spielt wie die Allgemeinmedizin eine wichtige Rolle in der Primärversorgung. Eine wichtige Schnittstelle von Psychiatrie/Psychosomatik und Allgemeinmedizin bildet die psychosomatische Grundversorgung (Wirsching und Fritzsche 1997). Die Forschungsansätze in beiden Fächern sind teilweise sozialwissenschaftlich orientiert.

Die Adressaten der Studie wurden bewusst anhand dieser Kriterien ausgewählt. Die Untersuchung erhebt daher nicht den Anspruch, ein repräsentatives Bild der Meinung aller medizinischen Hochschullehrer zu zeichnen. Sie kann aber durch Einfluss von wichtigen Meinungsbildnern einen Trend und möglicherweise relevante Gruppenunterschiede aufzeigen (Friedrichs 1990, Roth 1993).

Die Namen und Adressen der Abteilungsvorstände und Dekane wurden aus den (zumeist im Internet verfügbaren) Vorlesungsverzeichnissen des Wintersemesters 1998/99 der 37 medizinischen Hochschulen entnommen. Die einzelnen Universitäten sind im Anhang 8.1 angeführt. Unklarheiten im Fall von nicht oder nur kommissarisch besetzten Posten wurden telefonisch geklärt. Zum Zeitpunkt der Befragung befanden sich die Abteilungen Gynäkologie und Pädiatrie am Klinikum der Universität Regensburg im Aufbau (Dekanat der Universität Regensburg 1999). Die 
Lehrkrankenhäuser der Universität übernahmen die Lehrverpflichtung. Daher beschränkt sich der Adressatenkreis an der Universität Regensburg auf den Dekan und die ärztlichen Direktoren der Chirurgie, Inneren Medizin und Psychiatrie/Psychosomatik.

\subsection{Fragebogen}

Der Fragebogen (Anhang 8.2) gliederte sich inhaltlich in fünf Themenblöcke:

1. Allgemeine Bewertung der Allgemeinmedizin

Die Befragten konnten die Rolle der Allgemeinmedizin im Gesundheitswesen, die Qualifikation der Allgemeinärzte und die Besonderheiten der allgemeinmedizinischen Krankenversorgung standardisiert bewerten. Dadurch sollte die allgemeine Einstellung zur Allgemeinmedizin gemessen werden. Eine Zustimmung zu den Aussagen in Frage 1, 3, 4, 5 und eine Ablehnung bei Frage 2, 6, 7 wurden als positive Haltung zur Allgemeinmedizin gewertet.

2. Bewertung allgemeinmedizinischer Forschung und Lehre

Die Einstellung wurde als positiv bewertet, wenn die Wichtigkeit bzw. Qualität allgemeinmedizinischer Forschung und Lehre als höherwertig oder anderen Fächern vergleichbar bewertet wurde. Eine zustimmende Antwort bei Frage 8 bis 10 und Frage 12 bis 17 sowie eine ablehnende Antwort bei Frage 11 galt ebenfalls als Indiz für eine positive Einstellung zur Allgemeinmedizin.

3. Bewertung zukünftiger Inhalte des Medizinstudiums unter besonderer Berücksichtigung allgemeinmedizinischer Themen

In den Fragen 18 bis 30 konnten die Befragten neue Studieninhalte, denen der Wissenschaftsrat eine wichtige Position in einem reformierten Medizinstudium beimisst, beurteilen. Zur Bewertung standen vor allem allgemeinmedizinisch relevante Themen wie Geriatrie, Familienmedizin, ambulante Versorgung, Therapie chronischer Krankheiten, rationale Arzneitherapie und der Einsatz von Computern in Ausbildung und Praxis.

4. Fragen zur Allgemeinmedizin an der betreffenden Universität Hier interessierte die Organisationsform der lokalen Abteilung für Allgemeinmedizin, die Zufriedenheit des Befragten mit dieser Lösung und die Frage nach persönlicher Bekanntschaft mit dem Abteilungsdirektor. Weiterhin wurde nach 
gemeinsamen Projekten der Abteilung des Adressaten mit der universitären Allgemeinmedizin und niedergelassenen Allgemeinärzten gefragt. Im positiven Fall war der Teilnehmer gebeten, das oder die Projekte stichpunktartig aufzuführen. Abschließend sollten persönliche Erfahrungen mit der universitären und praktischen Allgemeinmedizin sowie die Relevanz des Faches an den Universitäten bewertet werden.

5. Soziodemographische Daten

Erfragt wurden Geschlecht und Alter, das Bundesland der betreffenden Universität, die Klinikzugehörigkeit bzw. Status des Befragten und eventuelle Arbeitszeiten in der Allgemeinmedizin.

Für die Studie wurden 45 standardisierte Fragen erarbeitet. Der Umfang des Fragebogens belief sich auf vier Druckseiten. Er enthielt drei unterschiedliche Frageund Antworttypen: 1. Bewertung von Aussagen anhand einer 5-stufigen Likert-Skalierung (z.B. Frage 1), 2. Alternativfragen (z.B. Frage 38), 3. halboffene Fragen (z.B. Frage 34).

\subsection{Durchführung der Untersuchung}

Zur Pilotierung des Fragebogens erklärten sich drei Abteilungsdirektoren der Universität Göttingen bereit. Aufgrund ihrer positiven Rückmeldung (vollständig ausgefüllte Fragebögen; keine Kritik auf Nachfrage) wurde der Fragebogen in seiner Form beibehalten. Dem Standard der empirischen Sozialforschung entsprechend (Bortz und Döring 1995) wurden die drei Testpersonen von der Hauptstudie ausgeschlossen.

Ende Juni 1999 wurden 599 Fragebögen mit einem persönlichen Anschreiben des Direktors der Abteilung Allgemeinmedizin der Universität Göttingen postalisch verschickt. Ein Freiumschlag zur Rücksendung lag bei.

\subsection{Datenanalyse}

Die Analyse der Daten erfolgte mit dem Statistikprogramm SAS in der Version 8.1 (Bärlocher 1999, Benninghaus 1998, SAS Institute 2000, Stokes et al.1995).

Zunächst wurde eine deskriptive, univariate Auswertung durchgeführt, deren Ergebnisse in Anhang $\mathbf{8 . 3}$ dargestellt sind. Zur übersichtlicheren Darstellung der 
Daten wurden Items mit einer Likert-Skalierung dichotomisiert. Galt die Zustimmung zu einer Frage als positive Haltung zur Allgemeinmedizin (Fragen 1, 3, 4 , 5, 10, 12, 15-17), wurden die zustimmenden gegen die neutralen und ablehnenden Befragten verglichen. Galt die Ablehnung einer Aussage als positive Haltung (Frage 2, 6, 7, 11) wurden ablehnende gegen neutrale und zustimmende Befragte verglichen. Im Fragenkomplex 18 bis 30 wurden die Antworten „sehr wichtig“ und „wichtig“ (als „wichtig“) gegen die neutralen bzw. negativen Positionen ausgewertet. Bei den Fragen 8, 9, 13 und 14 antworteten viele Befragte „neutral“ und „kann ich nicht beurteilen“, so dass sich die Bildung von vier Klassen („Zustimmung“, „Ablehnung“, „Neutral“ und „keine Angabe“) anbot. Antworten auf die Fragen 36 und 37 wurden zu drei Gruppen („positive Erfahrungen“, „,neutrale und negative Erfahrungen“ und „keine Erfahrung“) zusammengefasst. Ebenfalls vereinfacht wurden die Angaben zu Arbeitszeiten in der Allgemeinmedizin. Gruppe 1 schloss diejenigen ein, die bis zu einem Jahr („wenig Erfahrung“), und Gruppe 2 diejenigen, die länger als ein Jahr („längere Erfahrung“) in der Allgemeinmedizin gearbeitet haben. Zur übersichtlicheren Darstellung wurden die Altersangaben in vier Altersklassen unterteilt. Die Bundesländer wurden den Kategorien „alte Länder“, „neue Länder“ und „Berlin“ zugeordnet.

Im zweiten Analyseschritt erfolgte eine bivariate Betrachtung der Ergebnisse. Aussagen der Befragten zu Bedeutung und Qualität der Allgemeinmedizin wurden in Beziehung zur Klinikzugehörigkeit, zum Arbeitsort in alten bzw. neuen Bundesländern sowie zur persönlichen Bekanntschaft mit dem Leiter der Abteilung Allgemeinmedizin gesetzt. Zur besseren Übersicht beschränkt sich die Darstellung der Ergebnisse auf einige ausgewählte Fragen.

In einem dritten Schritt wurden die Daten multivariat ausgewertet (Ely et al. 1996, Abrahamson 1988). Hierdurch sollten die Einflussfaktoren bestimmt werden, die sich positiv bzw. negativ auf die Einstellung zur Allgemeinmedizin auswirkten. Als Verfahren wurde die multiple logistische Regression gewählt. Effektmaße zur Abschätzung der relativen Einflussgröße einzelner Faktoren waren die adjustierten Odds Ratios (OR) mit ihren 95\%-Konfidenzintervallen (KI). 
Zur effizienteren Bearbeitung wurden die Bewertungen der Themenbereiche „Bedeutung primärärztlicher Versorgung“ und „Bedeutung und Qualität allgemeinmedizinischer Forschung und Lehre" summiert.

In Zusammenschau aller Einzelwertungen wurden für den ersten Bereich die Fragen 2, 4 und 7 als besonders repräsentativ ausgewählt, für den zweiten Teil die Fragen 8, 10, 13, 14 und 16. Die Definitionen aus Kapitel 4.3, die in Anlehnung an die Studie von Block et al. (1996) erarbeitet wurden, legten es nahe, einen Summenwert von unter $7 \mathrm{im}$ ersten Fragenblock als positive Einstellung zur primärärztlichen Versorgung zu deuten. Ein Summenwert unter 14 galt im zweiten Fragenblock als positive Einstellung zur universitären Allgemeinmedizin. Eine Validierung dieser Einteilung erfolgte anhand einer Stichprobe von 50 zufällig ausgewählten Fragebogen. Alle Fragen der Komplexe 1 und 2 wurden nach dem obigen Prinzip summiert und anhand ihres Summenwertes entsprechend der Definitionen aus Kapitel 4.3 in jeweils zwei Gruppen („positiv“/,negativ“) eingeteilt. Für die Bewertung „primärärztlicher Versorgung" zeigte der Score eine Sensitivität von 0,83 und eine Spezifität von 0,92 . Im Score „wissenschaftliche Allgemeinmedizin“ ergab sich eine Sensitivität von 0,75 und eine Spezifität von 0,89 . 


\section{Ergebnisse}

\subsection{Teilnahme}

Es wurden insgesamt 599 Fragebogen verschickt. Drei Fragebogen kamen zurück, weil ein Lehrstuhl nicht besetzt und zwei Lehrstühle im Berufungsverfahren waren. Die bereinigte Grundgesamtheit betrug also $n=596$. Drei Wochen nach Aussendung waren 343 Fragebogen eingegangen. Durch eine telefonische und eine schriftliche Nachfrage nach weiteren drei Wochen wurden nochmals 132 Fragebogen zurückgesandt; darunter einer mit einer schriftlichen Begründung für die Verweigerung. Somit lag die Teilnahmerate bei $79 \%$ (474/596).

\subsection{Beschreibung der Stichprobe}

Die Grundauswertung der Daten ist im Anhang 8.3 dargestellt.

94,4\% der Teilnehmer waren männlich, 5,6\% weiblich. Das Altersspektrum lag zwischen 30 und 68 Jahren; im Mittel bei 53 Jahren (Median ebenfalls 53 Jahre). Die größte Gruppe repräsentierten die 50 bis 59-Jährigen.

Tabelle 1 Stichprobe nach Klinikzugehörigkeit

\begin{tabular}{lcc}
\hline Klinik & $\mathbf{n}^{*}$ & (\%) \\
\hline Chirurgie & 120 & $(25,7)$ \\
Gynäkologie & 42 & $(9,0)$ \\
Innere & 126 & $(27,0)$ \\
Pädiatrie & 77 & $(16,5)$ \\
Psychiatrie/Psychosomatik & 76 & $(16,3)$ \\
Dekan & 26 & $(5,6)$ \\
\hline
\end{tabular}

* Basis $n=467$

Drei Viertel (335/450) der Klinikdirektoren oder Dekane waren an Universitäten in den alten Bundesländern tätig, 18\% in den neuen Bundesländern und 34 in Berlin (Ost und West). Die Hälfte der Teilnehmenden stammte aus den großen Fächern Innere Medizin und Chirurgie, knapp 6\% waren Dekane (Tabelle 1). Arbeitserfahrungen in der Allgemeinmedizin hatten nach eigenen Angaben 186 Befragte (40\%), von diesen waren 35 länger als ein Jahr in der Allgemeinmedizin tätig. 


\subsection{Bewertung allgemeinmedizinischer Krankenversorgung, Forschung und Lehre}

Die Bewertung allgemeinmedizinisch/primärärztlicher Themen fiel insgesamt positiv für die Allgemeinmedizin aus (Tabelle 2): Fast $90 \%$ der Befragten sahen in Allgemeinärzten einen wichtigen Baustein des deutschen Gesundheitssystems und knapp zwei Drittel hielten Spezialisten für weniger geeignet als Allgemeinärzte die primärärztliche Versorgung sicherzustellen. $71,3 \%$ glaubten, dass ihre allgemeinmedizinischen Kollegen mit einer größeren therapeutischen und diagnostischen Unsicherheit arbeiten müssen als sie selbst. Zwei Drittel der Befragten sahen Allgemeinärzte stärker als Spezialisten durch die Wünsche des Patienten beeinflusst. Die meisten Direktoren und Dekane wiesen den Vorwurf zurück, Allgemeinärzte seien nur "Schmalspurmediziner".

Eine Abweichung von dieser betont positiven Beurteilung der Allgemeinmedizin zeigte sich in zwei Fragen: Nur jeder zweite hielt den Allgemeinarzt für besonders geeignet, chronisch kranke Patienten zu betreuen. Den Vorwurf der Unwissenschaftlichkeit in der allgemeinmedizinischen Entscheidungsfindung wiesen lediglich $26 \%$ der Teilnehmer zurück.

\section{Tabelle 2 Bewertung primärmedizinischer Versorgung und Funktionen}

Positive Aussage *

\begin{tabular}{|c|c|}
\hline & $\mathrm{n}^{* *}(\%)$ \\
\hline Allgemeinärzte arbeiten mit einer größeren therapeutischen Unsicherheit. & $335(71,3)$ \\
\hline $\begin{array}{l}\text { Allgemeinärzte stellen die primärärztliche Versorgung besser als } \\
\text { Spezialisten sicher. }\end{array}$ & $301(64,0)$ \\
\hline Allgemeinärzte haben eine wichtige Aufgabe im Gesundheitssystem. & $407(86,8)$ \\
\hline Allgemeinärzte sind besonders befähigt, chronisch Kranke zu betreuen. & $238(50,6)$ \\
\hline Allgemeinärzte sind stärker durch Patientenwünsche beeinflusst. & $312(66,7)$ \\
\hline Allgemeinmedizinische Entscheidungsfindung ist nicht unwissenschaftlich. & $121(25,8)$ \\
\hline Allgemeinärzte sind keine „Schmalspurmediziner“. & $373(80,0)$ \\
\hline
\end{tabular}

* Für die Definition positive Aussage gelten die in Kapitel 4.3 angegebenen Kriterien.

** Die Zahl der Antwortenden schwankte zwischen $n=466$ und $n=470$.

\section{Zustimmung}

$301(64,0)$

$407(86,8)$

$373(80,0)$ 
Bedeutung und vor allem Qualität allgemeinmedizinischer Forschung und Lehre wurden eher zurückhaltend beurteilt (Tabelle 3): Fast die Hälfte der Befragten hielt die Forschung in der Allgemeinmedizin für wichtiger oder genauso wichtig wie die Forschung anderer Fächer. Nicht einmal jeder Fünfte beurteilte die Qualität dieser Forschung als besser oder gleichwertig im Vergleich zu anderen Forschungsbereichen. Lediglich ein Viertel aller Antwortenden widersprach dem Vorwurf, Allgemeinärzte hätten kein Interesse an Forschung teilzunehmen. Eine gleichberechtigte Berücksichtigung sozialwissenschaftlicher Forschungsmethoden im Medizinstudium unterstützte die Hälfte der Teilnehmer.

Ähnlich gestaltete sich die Einschätzung der Lehre. Während immerhin $76 \%$ der Hochschullehrer von der Bedeutung allgemeinmedizinischer Lehre überzeugt waren, bewertete nur jeder Dritte die Qualität als besser oder anderen Fächern vergleichbar. Die inhaltliche und methodische Eigenständigkeit des Faches Allgemeinmedizin akzeptierten knapp 60\% der Befragten.

\section{Tabelle 3 Einstellung zur universitären Allgemeinmedizin}

Positive Aussage*

Zustimmung

$\mathrm{n}^{\star *}(\%)$

Die Bedeutung allgemeinmedizinischer Forschung ist der anderer Fächer vergleichbar.

Die Qualität allgemeinmedizinischer Forschung ist der anderer Fächer vergleichbar.

Sozialwissenschaftliche und naturwissenschaftliche Methoden sollten im Studium gleichberechtigt sein.

Allgemeinmedizinische Forschungsergebnisse sind auch für andere Fächer relevant.

Allgemeinärzte sind an Forschung interessiert.

Die Bedeutung allgemeinmedizinischer Lehre ist der anderer Fächer vergleichbar.

Die Qualität allgemeinmedizinischer Lehre ist der anderer Fächer vergleichbar.

Allgemeinmedizinische Lehre ist methodisch eigenständig.

Allgemeinmedizinische Lehre ist inhaltlich eigenständig.

$276(59,0)$

* Für die Definition Positive Aussage gelten die in Kapitel 4.3 angeführten Kriterien.

** Die Zahl der Antwortenden schwankte zwischen $n=458$ und $n=468$. 
Die meisten der in Tabelle 4 aufgeführten Themen betrachtete die überwiegende Mehrheit der antwortenden Hochschullehrer als wichtige Inhalte des zukünftigen Medizinstudiums. Lediglich drei Themen waren weniger als $70 \%$ der Befragten eine verstärkte Integration ins Medizinstudium wert: Familienmedizin und der Einsatz des PC für Literaturrecherche und zur Wissensvermittlung.

\section{Tabelle 4 Bedeutung zukünftiger Ausbildungsinhalte}

\begin{tabular}{ll}
\hline Ausbildungsinhalt* & $\mathbf{n}^{\star *}(\%)$ \\
\hline Geriatrie & $408(87,6)$ \\
Therapie chronischer Krankheiten & $444(94,5)$ \\
Multimorbidität & $404(86,3)$ \\
Familienmedizin & $276(59,8)$ \\
Ambulante Versorgung & $393(84,5)$ \\
Rationale Arzneimitteltherapie & $430(91,9)$ \\
Kenntnisse des Sozialsystems & $350(74,3)$ \\
Gesundheitsförderung & $369(79,0)$ \\
Gesprächsführung & $396(84,6)$ \\
PC für Literaturrecherche & $310(66,2)$ \\
PC für Wissensvermittlung & $309(66,0)$ \\
PC für Dokumentation u. Abrechnung & $364(77,8)$ \\
Ökonomische Konsequenzen ärztlichen Handelns & $397(84,8)$ \\
\hline
\end{tabular}

* Angegeben ist die Zahl (\%) derjenigen, die diese Inhalte für (sehr) wichtig erachten.

** Die Zahl der Antwortenden schwankte zwischen $n=461$ und $n=471$.

\subsection{Universitäre Institutionalisierung der Allgemeinmedizin}

Die meisten der befragten Hochschullehrer gaben an, die Allgemeinmedizin an ihrer Universität sei durch Lehraufträge oder einen eigenen Lehrstuhl repräsentiert. Jeder Zehnte wusste hierüber nichts Genaueres (Tabelle 6). Von den Teilnehmern, denen die Institutionalisierung der Allgemeinmedizin an der Hochschule bekannt war, waren $60 \%$ mit dieser Lösung zufrieden, etwa ein Viertel der Befragten (97/417) lehnte dieses Modell ab. An Universitäten mit eigenem Lehrstuhl war die überwiegende Mehrheit der Professoren (104/123) mit der Institutionalisierung zufrieden. Wurde die Allgemeinmedizin an der Hochschule durch Lehraufträge sichergestellt, war es nur knapp die Hälfte der Befragten (100/213). 60\% der Teilnehmer kannten den Direktor 
der Abteilung Allgemeinmedizin bzw. den Sprecher des Lehrauftrages persönlich. Ein Viertel der Befragten berichtete von gemeinsamen Projekten mit der universitären Allgemeinmedizin, die oft im Bereich Lehre und Klinische Studien angesiedelt waren. Die gemeinsamen Projekte mit den niedergelassenen Allgemeinärzten, die in $37 \%$ der Fälle bestanden, waren meist Bestandteil von Fort- und Weiterbildung.

Tabelle 5 Universitäre Organisationsform der Allgemeinmedizin; nach Angaben der Teilnehmer

\begin{tabular}{lc}
\hline Institutionalisierung & $\mathbf{n}^{\star *}(\%)$ \\
\hline Lehrstuhl & $123(26,4)$ \\
Lehrbereich & $63(13,5)$ \\
Lehrauftrag & $215(46,1)$ \\
Andere & $15(3,2)$ \\
Unbekannt & $50(10,7)$ \\
\hline Es besteht eine persönliche Bekanntschaft mit dem Leiter der Allgemeinmedizin. & $275(60,4)$ \\
Es existieren gemeinsame Projekte mit der universitären Allgemeinmedizin. & $115(26,1)$ \\
Es existieren Kontakte zur regionalen Allgemeinmedizin. & $174(37,4)$ \\
\hline
\end{tabular}

** Die Zahl der Antwortenden schwankte zwischen $n=444$ und $n=468$.

Die persönlichen Erfahrungen mit der universitären Allgemeinmedizin beurteilte etwa ein Drittel als positiv bzw. negativ, 182 Befragte enthielten sich einer Bewertung, da sie keinerlei Erfahrungen mit der Allgemeinmedizin an ihrer Universität hatten. Eine Stärkung der universitären Allgemeinmedizin befürworteten zwei Drittel der Befragten, 82,8\% hielten die Allgemeinmedizin im Studium für unverzichtbar, einen allgemeinmedizinisch interessierten Studenten würden 72,5\% der Hochschullehrer in seinem Berufswunsch „Hausarzt“ unterstützen (Tabelle 7).

\section{Tabelle 6 Unterstützung der universitären Allgemeinmedizin}

\begin{tabular}{lc}
\hline Aussage & $\begin{array}{c}\text { Zustimmung } \\
\mathbf{n}^{\star *} \mathbf{\%}\end{array}$ \\
\hline Befürwortung einer Stärkung der Allgemeinmedizin im Studium & $314(67,1)$ \\
Unverzichtbarkeit der Allgemeinmedizin im Studium & $389(82,8)$ \\
Unterstützung von Studenten mit Berufsziel Allgemeinmedizin & $335(72,5)$ \\
\hline
\end{tabular}

** Die Zahl der Antwortenden schwankte zwischen $n=462$ und $n=470$. 


\subsection{Bewertung der Allgemeinmedizin aus Sicht der Kliniken}

Unterschiede in der Beurteilung der Allgemeinmedizin wurden besonders zwischen Innerer Medizin und Psychiatrie/Psychosomatik deutlich, wobei die Befragten aus Psychiatrie/Psychosomatik und zumeist auch die Dekane der Allgemeinmedizin gegenüber vergleichsweise positiv eingestellt waren. In der Inneren Medizin dominierten kritischere Einschätzungen. Beispielsweise wurde die primärärztliche Versorgung von $40 \%$ der Chirurgen, $47 \%$ der Befragten aus Psychiatrie/Psychosomatik und $50 \%$ der Dekane positiv eingeschätzt, jedoch nur von $27 \%$ der internistischen Abteilungsleiter (Tabelle 8). Von diesen hatten auch nur $18 \%$ eine positive Meinung über die wissenschaftliche Qualität der Allgemeinmedizin in Forschung und Lehre, dagegen über die Hälfte der Direktoren aus Psychiatrie und Psychosomatik. Am häufigsten hatten die Dekane Kontakt mit der universitären Allgemeinmedizin: Fast alle kannten den Leiter oder Sprecher der allgemeinmedizinischen Abteilung bzw. Lehrbeauftragten. Weniger als die Hälfte aller Befragten hatte positive Erfahrungen mit der universitären Allgemeinmedizin gemacht, am häufigsten noch die Dekane.

80\% der Abteilungsvorsteher der Psychiatrie/Psychosomatik wollten die Allgemeinmedizin im Studium gestärkt sehen, bei den Dekanen waren es immerhin noch $70 \%$, bei den Chefärzten der Inneren Medizin lediglich die Hälfte der Befragten.

Zur einfacheren Darstellung der Ergebnisse wurde die Bewertung primärmedizinischer Versorgung und Funktionen und die Einstellung zur universitären Allgemeinmedizin anhand der Klinikzugehörigkeit zu einer gemeinsamen Variable zusammengefasst (s. Kapitel 4.5). Die vollständige Auswertung beider Themenbereiche ist als Anhang 8.4 und 8.5 einzusehen. 
Tabelle 7 Bewertungen der akademischen Allgemeinmedizin; nach Klinikzugehörigkeit

\begin{tabular}{|c|c|c|c|c|c|c|}
\hline Kriterien & $\begin{array}{l}\text { Chirurgie } \\
\qquad(n=120)\end{array}$ & $\begin{array}{l}\text { Gynäko- } \\
\text { logie } \\
(n=42)\end{array}$ & $\begin{array}{c}\text { Innere } \\
\text { Medizin } \\
(n=126)\end{array}$ & $\begin{array}{c}\text { Pädiatrie } \\
\text { (n=77) }\end{array}$ & $\begin{array}{l}\text { Psychiatrie } \\
\text { u. Psycho- } \\
\text { somatik } \\
(n=76)\end{array}$ & $\begin{array}{l}\text { Dekane } \\
(n=26)\end{array}$ \\
\hline $\begin{array}{l}\text { Positive Haltung zur } \\
\text { primärärztlichen } \\
\text { Versorgung }^{*}\end{array}$ & $40,0 \%$ & $40,5 \%$ & $27,0 \%$ & $26 \%$ & $47,4 \%$ & $50,0 \%$ \\
\hline $\begin{array}{l}\text { Positive Haltung zur } \\
\text { wissenschaftlichen } \\
\text { Allgemeinmedizin* }\end{array}$ & $17,5 \%$ & $59,5 \%$ & $18,3 \%$ & $37,7 \%$ & $55,3 \%$ & $38,5 \%$ \\
\hline $\begin{array}{l}\text { Bekanntschaft mit } \\
\text { dem Leiter der } \\
\text { Allgemeinmedizin }\end{array}$ & $56 \%$ & $41 \%$ & $75 \%$ & $58,7 \%$ & $47,1 \%$ & $88,5 \%$ \\
\hline $\begin{array}{l}\text { Zufriedenheit mit der } \\
\text { Institutionalisierung }\end{array}$ & $66 \%$ & $54,3 \%$ & $65,5 \%$ & $62 \%$ & $45,5 \%$ & $50 \%$ \\
\hline $\begin{array}{l}\text { Gemeinsame } \\
\text { Projekte mit der } \\
\text { Allgemeinmedizin }\end{array}$ & $22,0 \%$ & $22 \%$ & $28,0 \%$ & $13,5 \%$ & $30,0 \%$ & - \\
\hline $\begin{array}{l}\text { Positive } \\
\text { Erfahrungen mit der } \\
\text { universitären } \\
\text { Allgemeinmedizin }\end{array}$ & $27,5 \%$ & $31 \%$ & $37,9 \%$ & $23,4 \%$ & $32,9 \%$ & $46,2 \%$ \\
\hline $\begin{array}{l}\text { Stärkung der } \\
\text { Allgemeinmedizin }\end{array}$ & $66,4 \%$ & $78,6 \%$ & $53,2 \%$ & $70,1 \%$ & $80,3 \%$ & $69,2 \%$ \\
\hline
\end{tabular}

* Für die Definition Positive Aussage gelten die in Kapitel 4.3 angeführten Kriterien.

\subsection{Bewertung der Allgemeinmedizin in Abhängigkeit vom Arbeitsort in alten und neuen Bundesländern}

Unterschiede im Antwortverhalten der Chefärzte und Dekane in alten und neuen Bundesländern bezogen sich besonders auf Bereiche, die die Institutionalisierung der Allgemeinmedizin an den Hochschulen betreffen. In den neuen Bundesländern kannte die Hälfte der Antwortenden den Leiter der Allgemeinmedizin persönlich, in den alten Ländern waren es $62 \%$. Waren hier genauso viele Befragte zufrieden mit der Institutionalisierung der Allgemeinmedizin, teilten im Osten nur $43 \%$ diese Meinung. Gemeinsame Projekte überwogen ebenfalls in den alten Bundesländern ( $27 \%$ vs $19 \%$ ). Etwa doppelt so viele Teilnehmer aus den alten Bundesländern hatten positive Erfahrungen mit der universitären Allgemeinmedizin (36\% vs $18 \%$ ). 
Vergleichsweise schlechter bewerteten die Chefärzte und Dekane aus den östlichen Bundesländern die primärärztliche Versorgung: Kaum mehr als ein Viertel schätzte sie positiv ein, jedoch knapp 40\% der Befragten aus den westlichen Bundesländern.

Die Bewertung der wissenschaftlichen Allgemeinmedizin und Befürwortung einer Stärkung der Allgemeinmedizin im Studium wurden ebenso wie eine Unterstützung von Studenten mit allgemeinmedizinischem Berufsziel überregional einheitlich beurteilt.

Tabelle 8 Bewertung der akademischen Allgemeinmedizin; nach Herkunft aus alten bzw. neuen Ländern

\begin{tabular}{lcc}
\hline & \multicolumn{2}{c}{ Chefärzte und Dekane aus den } \\
Kriterium & $\begin{array}{c}\text { alten Bundesländern } \\
\text { (inklusive Berlin) } \\
\text { (n=369) }\end{array}$ & $\begin{array}{c}\text { neuen Bundesländern } \\
\text { (n= 81) }\end{array}$ \\
\hline $\begin{array}{l}\text { Positive Bewertung } \\
\text { primärärztlicher Versorgung* }\end{array}$ & $39 \%(144)$ & $27,2 \%(22)$ \\
$\begin{array}{l}\text { Positive Bewertung wissenschaftlicher } \\
\text { Allgemeinmedizin* }\end{array}$ & $29 \%(107)$ & $29,6 \%(24)$ \\
$\begin{array}{l}\text { Bekanntschaft mit dem } \\
\text { Leiter der Allgemeinmedizin }\end{array}$ & $62,2 \%(222)$ & $50 \%(38)$ \\
Zufriedenheit mit Institutionalisierung & $62,3 \%(190)$ & $43 \%(28)$ \\
$\begin{array}{l}\text { Gemeinsame Projekte mit der } \\
\text { Allgemeinmedizin }\end{array}$ & $27,4 \%(94)$ & $18,8 \%(15)$ \\
$\begin{array}{l}\text { Positive Erfahrungen mit der } \\
\text { universitären Allgemeinmedizin } \\
\text { Unterstützung eines Studenten } \\
\text { mit Berufsziel Allgemeinmedizin }\end{array}$ & $35,6 \%(131)$ & $17,7 \%(14)$ \\
Stärkung der Allgemeinmedizin & $73,7 \%(266)$ & $70 \%(56)$ \\
\hline
\end{tabular}

* Für die Definition Positive Aussage gelten die in Kapitel 4.3 angeführten Kriterien.

\subsection{Bewertung der akademischen Allgemeinmedizin in}

\section{Abhängigkeit von der persönlichen Bekanntschaft mit dem Vorstand der Abteilung Allgemeinmedizin}

Professoren, die persönlich mit dem Leiter der Abteilung Allgemeinmedizin bekannt sind, bewerteten die primärärztliche Versorgung bzw. die wissenschaftliche Allgemeinmedizin gleich häufig positiv wie ihre Kollegen ohne persönliche Kontakte 
(je 37\% bzw. 29\% der Befragten). Die persönliche Bekanntschaft beeinflusste allerdings die Einschätzung der Institutionalisierung der Allgemeinmedizin an der jeweiligen Hochschule: Zwei Drittel der Hochschullehrer mit persönlichen Kontakten waren mit der Institutionalisierung der Allgemeinmedizin an ihrer Hochschule zufrieden, in der Vergleichsgruppe waren es weniger als die Hälfte (69\% vs 43\%). Lediglich 15\% der Abteilungsdirektoren ohne persönliche Kontakte (44\% der Leiter mit persönlichen Kontakten) berichteten über positive Erfahrungen mit der universitären Allgemeinmedizin. Allerdings unterstützten letztere die Stärkung der Allgemeinmedizin im Studium häufiger als Ihre Kollegen (71\% vs 65\%). Über drei Viertel der Professoren mit persönlichen Verbindungen in die Allgemeinmedizin würden einen Studenten mit Berufsziel Allgemeinmedizin unterstützen, in der Vergleichsgruppe waren es deutlich weniger (78\% vs $65 \%)$.

Tabelle 9 Bewertung der Allgemeinmedizin; nach persönlicher

Bekanntschaft mit dem Leiter der Abteilung Allgemeinmedizin

\begin{tabular}{|c|c|c|}
\hline \multirow[b]{2}{*}{ Kriterium } & \multicolumn{2}{|c|}{ Chefärzte und Dekane } \\
\hline & $\begin{array}{c}\text { mit } \\
\text { persönlichem Kontakt } \\
(n=275)\end{array}$ & $\begin{array}{c}\text { ohne } \\
\text { persönlichen Kontakt } \\
(n=180)\end{array}$ \\
\hline $\begin{array}{l}\text { Positive Bewertung } \\
\text { primärärztlicher Versorgung }\end{array}$ & $37,5 \%(103)$ & $36,7 \%(66)$ \\
\hline $\begin{array}{l}\text { Positive Bewertung } \\
\text { wissenschaftlicher Allgemeinmedizin* }\end{array}$ & $29,5 \%(81)$ & $29,4 \%(53)$ \\
\hline Zufriedenheit mit Institutionalisierung & $68,6 \%(188)$ & $42,9 \%(57)$ \\
\hline $\begin{array}{l}\text { Gemeinsame Projekte } \\
\text { mit der Allgemeinmedizin }\end{array}$ & $36,3 \%(93)$ & $11,3 \%(19)$ \\
\hline $\begin{array}{l}\text { Positive Erfahrungen mit der } \\
\text { universitären Allgemeinmedizin }\end{array}$ & $44,3 \%(121)$ & $14,7 \%(26)$ \\
\hline $\begin{array}{l}\text { Unterstützung eines Studenten } \\
\text { mit Berufsziel Allgemeinmedizin }\end{array}$ & $78 \%(209)$ & $64,8 \%(114)$ \\
\hline Stärkung der Allgemeinmedizin & $64,6 \%(175)$ & $71 \%(127)$ \\
\hline
\end{tabular}

* Für die Definition Positive Aussage gelten die in Kapitel 4.3 angeführten Kriterien. 


\subsection{Einflussfaktoren auf die Entscheidung}

\section{„Stärkung der Allgemeinmedizin im Studium“}

In einer abschließenden Analyse sollte untersucht werden, welche Faktoren eine positive Grundhaltung zur Allgemeinmedizin fördern. Zielkriterium war dabei die Bereitschaft, die Allgemeinmedizin im Studium zu stärken. Prädiktoren (im statistischen Sinne) waren unter anderem die Einstellung zur Primärversorgung, die Bewertung der wissenschaftlichen Allgemeinmedizin, die Bekanntschaft mit dem Leiter der Allgemeinmedizin, eigene Arbeitserfahrung in der Allgemeinmedizin, das Alter und die Klinikzugehörigkeit (weitere Prädiktoren s. Tabelle 10). Der Einfluss dieser Prädiktoren auf die Befürwortung einer Stärkung der Allgemeinmedizin im Studium wird in Tabelle 10 durch Odds Ratios dargestellt, zunächst unadjustiert (linke Spalten), dann adjustiert, also multivariat kontrolliert (rechte Spalten). Zusätzlich zeigt Tabelle 11 das Ergebnis einer multivariaten Analyse mit Rückwärtsselektion signifikanter Variablen, um so das „Hintergrundrauschen“ nicht-signifikanter Variablen auszublenden. Tabelle 10 markiert als signifikante Einflussvariablen für die Bereitschaft, die Allgemeinmedizin im Studium zu stärken, die Einstellung zur Primärversorgung, die Einstellung zur wissenschaftlichen Allgemeinmedizin und die Zugehörigkeit zur Inneren Medizin. Die adjustierte Berechnung ergab ein fast identisches Bild: lediglich die Herkunft aus der Inneren Medizin verliert an Signifikanz.

In der abschließenden multivariaten Analyse mit Rückwärtsselektion wurden diese drei Variablen (inklusive der Klinikzugehörigkeit Innere Medizin) als signifikante Einflussfaktoren ermittelt. Für eine Stärkung entschieden sich besonders Abteilungsdirektoren mit einer positiven Einstellung zur wissenschaftlichen Allgemeinmedizin (OR 5,5; 95\%-KI 2,9-10,4) und diejenigen mit einer wohlwollenden Haltung zur primärärztlichen Versorgung (OR 2,6; 95\%-KI 1,6-4,3). Im Vergleich zu den Befragten aus der Psychiatrie war die Bereitschaft der Professoren aus der Inneren Medizin, die Allgemeinmedizin zu fördern, deutlich geringer (OR 0,48; 95\%-KI 0,3-0,8). 
Tabelle 10 Stärkung der Allgemeinmedizin im Studium - Einflussfaktoren

\begin{tabular}{|c|c|c|c|c|c|c|}
\hline \multirow[b]{3}{*}{ Variable } & \multicolumn{6}{|c|}{ Stärke der Einflussfaktoren } \\
\hline & \multirow[b]{2}{*}{ OR } & \multicolumn{3}{|l|}{ unadjustiert } & \multicolumn{2}{|l|}{ adjustiert } \\
\hline & & $\begin{array}{l}\text { 95\%-Konfidenz- } \\
\text { intervall }\end{array}$ & $\mathbf{p}$ & OR & $\begin{array}{l}\text { 95\%-Konfidenz- } \\
\text { intervall }\end{array}$ & $\mathbf{p}$ \\
\hline \multicolumn{7}{|c|}{ Einstellung zur Primärversorgung } \\
\hline - neutral & 1,0 & & & 1,0 & & \\
\hline - positiv & 3,39 & $2,16-5,32$ & $<0,001$ & 2,66 & $1,6-4,4$ & 0,002 \\
\hline \multicolumn{7}{|c|}{ Einstellung zur wissenschaftlichen Allgemeinmedizin } \\
\hline - neutral & 1,0 & & & 1,0 & & \\
\hline - positiv & 6,71 & $3,71-12,15$ & $<0,001$ & 6,01 & $3,09-11,6$ & $<0,001$ \\
\hline \multicolumn{7}{|l|}{ Alter } \\
\hline - > 60 Jahre & 1,0 & & & 1,0 & & \\
\hline - 30-59 Jahre & 1,01 & $0,62-1,62$ & 0,9639 & 0,97 & $0,94-1,00$ & 0,0837 \\
\hline \multicolumn{7}{|c|}{ Arbeitserfahrung in der Allgemeinmedizin } \\
\hline- nein & 1,0 & & & 1,0 & & \\
\hline - ja & 0,72 & $0,49-1,06$ & 0,1026 & 0,64 & $0,40-1,02$ & 0,060 \\
\hline \multicolumn{7}{|c|}{ Erfahrungen mit der universitären Allgemeinmedizin } \\
\hline - neutral & 1,0 & & & 1,0 & & \\
\hline - positiv & 1,38 & $0,91-2,11$ & 0,1273 & 1,22 & $0,72-2,08$ & 0,4511 \\
\hline
\end{tabular}

Persönliche Bekanntschaft mit dem Leiter der Allgemeinmedizin

- nein

- ja

Gemeinsame Projekte

- nein

- ja

Bundesland

- neue Länder/Berlin

- alte Länder

Institutionalisierung

- andere Formen

- Lehrstuhl

\section{Klinik}

- Psychiatrie

- Gynäkologie

$1,93 \quad 0,90-4,16$

$1,21 \quad 0,71-2,06$

$1,13 \quad 0,48-2,67$

$0,95 \quad 0,61-1,48$ $0,43 \quad 0,28-0,65$

- Pädiatrie

- Dekan

- Chirurgie

- Innere

1,0

0,62

$0,36-1,06$

0,0810

1,0

$1,60 \quad 1,00-2,56$

1,0

$1,14 \quad 0,84-1,55$

1,0

$1,19 \quad 0,76-1,85$

0,4358

0,90

$0,53-1,54$

0,7221

\begin{tabular}{lll} 
& 1,0 & \\
0,0849 & 1,82 & $0,64-5,20$ \\
0,4723 & 0,96 & $0,40-2,26$ \\
0,7696 & 0,55 & $0,16-1,80$ \\
0,8375 & 0,92 & $0,42-2,00$ \\
0,0001 & 0,53 & $0,24-1,16$ \\
\hline
\end{tabular}

0,2584

0,9277

0,3263

0,8353

0,1159 
Tabelle 11 Stärkung der Allgemeinmedizin im Studium - Einflussfaktoren *

\begin{tabular}{llll}
\hline & \multicolumn{3}{c}{ Stärke des Einflussfaktors } \\
\cline { 2 - 4 } Variable & OR & $95 \%-K o n f i d e n z i n t e r v a l l$ & $p$
\end{tabular}

Einstellung zur primärärztlichen Versorgung

- neutral

1,0

- positiv

2,605

$1,59-4,25$

0,0001

Einstellung zur wissenschaftlichen Allgemeinmedizin

- neutral

1,0

- positiv

5,52

$2,94-10,35$

$<0,0001$

Klinik

- Psychiatrie

1,0

- Innere

0,48

$0,30-0,76$

0,0021

* Modell mit backward elimination 


\section{Diskussion}

Die vorliegende Arbeit untersucht die Haltung von Hochschullehrern an deutschen medizinischen Fakultäten zur akademischen Allgemeinmedizin. Die Mehrheit der Befragten sieht die Allgemeinmedizin als festen Bestandteil des universitären Alltags. Zwei Drittel befürworten eine Stärkung des Faches innerhalb des Curriculums. Auch die Rolle der Allgemeinmedizin in der Primärversorgung wird überwiegend positiv bewertet. Im Gegensatz hierzu steht die schlechte Beurteilung der Leistungen der Allgemeinmedizin in Forschung und Lehre.

\subsection{Methode}

Die deskriptiv-explorative Ausrichtung dieser Studie berücksichtigte die Tatsache, dass bislang im deutschsprachigen Raum keine wissenschaftlichen Datenerhebungen zu diesem Thema durchgeführt wurden. Sie erlaubte im Weiteren den Vergleich einzelner Subgruppen und eine detaillierte Betrachtung der Ergebnisse anhand einer multivariaten Analyse (Bortz 1984, Friedrichs 1990).

Adressaten der Studie waren die Dekane und Direktoren ausgewählter klinischer Abteilungen, die in einem besonderen Verhältnis zur Allgemeinmedizin stehen. Hierbei handelte es sich ausschließlich um Fächer der klinischen Studienabschnitte (Innere, Chirurgie, Pädiatrie, Psychiatrie, Gynäkologie). Die Zielgruppe spiegelt also nicht die tatsächliche Zusammensetzung der medizinischen Fakultäten wider und ist somit nicht repräsentativ für die akademische Medizin. Allerdings handelt es sich um eine Totalerhebung bei der definierten Gesamtheit, sodass eine valide Aussage für diese wichtigen klinischen Fächer getroffen und mögliche Trends aufgezeigt werden können. Da die Befragten in ihrer Funktion sowohl relevante Meinungsbildner als auch Entscheidungsträger in der Fakultät sind, ermöglichen die Ergebnisse einen Ein- und Ausblick auf die Fremdeinschätzung der Allgemeinmedizin an den deutschen Hochschulen.

Aufgrund der regionalen Streuung, der großen Anzahl der Adressaten und ihrer soziodemographischen Homogenität wurde die schriftliche Befragung mit einem standardisierten Fragebogen als Instrument gewählt (Bortz 1984, Friedrichs 1990, Roth 1993). Ein Hauptproblem schriftlicher Befragungen ist die Sicherstellung einer 
ausreichenden Rücklaufquote (Geyer und Siegrist 1998, Hippler 1985, Hippler 1988, Thoma und Zimmermann 1996). Die Entwicklung der „total design method“ (TDM) durch Dillman (Dillman 1978) erhöhte die Ausschöpfquoten schriftlicher Erhebungen signifikant (Porst 2001, Porst et al. 2001). Folgerichtig wird in der heutigen sozialwissenschaftlichen Forschung die schriftliche Befragung als dem Telefoninterview bzw. dem persönlich-mündlichen Interview gleichwertig eingestuft (Klein und Porst 2000).

Inhaltliche und formale Gestaltung dieser Untersuchung orientierten sich daher an den Kriterien der TDM. Die Pilotierung des Fragebogens erfolgte durch drei Professoren der Universität Göttingen, die entsprechend den Standards moderner Sozialforschung aus der Hauptstudie ausgeschlossen wurden (Bortz und Döring 1995). Aufgrund ihrer positiven Rückmeldungen wurde der Fragebogen unverändert beibehalten. Die Rücklaufquote der Hauptstudie lag trotz des ungünstigen Erhebungszeitpunkts (vorlesungsfreie Zeit im Sommersemester) bei $79 \%$, was als sehr gutes Ergebnis zu werten ist (z. B. Babbie 1995).

Ein Nachteil der postalischen Befragung ist die unkontrollierbare Erhebungssituation. Eine mögliche Delegation des Fragebogens an eine(n) Mitarbeiter(in) des Adressaten ist nicht ausgeschlossen. Als Hinweis hierauf kann die Differenz zwischen angeschriebenen $(n=11)$ und antwortenden $(n=26)$ Frauen gesehen werden. Deshalb wurde in dieser Studie - auch in Hinblick auf die geringe Anzahl teilnehmender Frauen - keine Auswertung nach Geschlecht durchgeführt. In der Literatur überwiegt die Ansicht, dass im Regelfall der Adressat auch derjenige ist, der den Fragebogen beantwortet (Brune et al. 1991).

In der Auswertung (Kapitel 5) fällt die auf den ersten Blick überwiegend positive Haltung zur Allgemeinmedizin auf. Eine Verzerrung der Ergebnisse durch eine Bewertung im Sinne der "sozialen Erwünschtheit“ („social desirability“; Edwards 1957) im Rahmen der aktuellen Diskussion um eine Aufwertung der Primärversorgung erscheint eher unwahrscheinlich, da es sich, wie bereits weiter oben ausgeführt, bei den Adressaten um Meinungsbildner und Entscheidungsträger handelt. Darüber hinaus wird dem Effekt der sozialen Erwünschtheit in postalischen Befragungen eine untergeordnete Rolle beigemessen (Rossi 1983, Klein und Porst 2000). 
Die Validität der gemachten Aussagen, z.B. durch Vergleich der Angabe zur Institutionalisierung der Allgemeinmedizin mit der tatsächlichen Etablierung an der jeweiligen Hochschule, wurde aus datenschutzrechtlichen Gründen nicht durchgeführt.

Die Datenanalyse verwendet Methoden der deskriptiven Statistik und der logistischen Regression. Die zur vereinfachten Bearbeitung und Darstellung eingeführten Summenscores wurden auf ihre Sensitivität und Spezifität getestet (Kapitel 4.5).

\subsection{Ergebnisse}

Insgesamt fiel die Bewertung der Allgemeinmedizin überwiegend positiv aus. Das Fach Allgemeinmedizin ist in den letzten 30 Jahren fester Bestandteil des medizinischen Curriculums geworden. Über 80 \% der Befragten hielten die Allgemeinmedizin im Studium für unverzichtbar und knapp drei Viertel würden eine Stärkung im Studium befürworten (Tabelle 5). Auch die primärärztliche Versorgung erzielte eine gute Bewertung: Knapp 90\% der Befragten sahen in der Allgemeinmedizin einen wichtigen Bestandteil des deutschen Gesundheitswesens und zwei Drittel waren überzeugt, dass Spezialisten die Primärversorgung nicht so gut wie Allgemeinärzte gewährleisten können (Tabelle 2). Diese positive Beurteilung unterscheidet die vorliegende Untersuchung deutlich von den bislang zu diesem Thema durchgeführten amerikanischen Studien (Block et al. 1996, Block et al. 1998, Zinn et al. 1998), die in der akademischen Medizin ein überwiegend "frostiges“ Klima gegenüber der Allgemeinmedizin fanden.

In einer 1996 veröffentlichten amerikanischen Studie (Block et al. 1996) wurden Dekane und Abteilungsdirektoren, Lehrende einzelner Fachgebiete, Weiterbildungsassistenten („residents“) und Medizinstudenten telefonisch zu Akzeptanz und Bewertung primärmedizinischer Inhalte befragt. Besonders Spezialisten bewerteten die Allgemeinmedizin negativ, wohingegen die Vertreter der "generalistischen" Fächer ${ }^{2}$ zumeist wohlwollend urteilten. Im Einzelnen wurden die für primärärztliche Aufgaben benötigte Qualifikation niedrig bewertet und die Fähigkeit von Allgemeinärzten, Patienten mit schweren Krankheiten adäquat zu betreuen, in Frage gestellt. Selbst

\footnotetext{
${ }^{2}$ Family Medicine, General Internal Medicine, General Pediatrics.
} 
für banale Behandlunsanlässe favorisierte fast ein Viertel der Befragten einen Spezialisten.

Aber auch in der vorliegenden Studie werden Kritikpunkte deutlich, die im folgenden näher erläutert werden.

\subsubsection{Der Allgemeinarzt und chronische Erkrankungen}

Lediglich $50 \%$ der Befragten hielten den Allgemeinarzt für den besten Arzt bei chronischen und fortschreitenden Erkrankungen. Dies ist bemerkenswert, da es sich gerade hier um typisch allgemeinmedizinische Aufgabenfelder handelt (Comberg 1994, DEGAM 2002a). Divergente Einschätzungen spiegeln sich auch in wissenschaftlichen Untersuchungen zur Qualität haus- und fachärztlicher Versorgung wider: So erzielten in der Studie von Harrold et al. (1999) Spezialisten bei bestimmten Erkrankungen bessere (Behandlungs)Ergebnisse als Allgemeinärzte. Insgesamt verfügten sie über ein größeres Wissen und setzten neue und effektive Behandlungsmethoden schneller in die Praxis um als ihre allgemeinmedizinischen Kollegen, z.B. bei der Behandlung von Asthmatikern. Bei anderen chronischen Erkrankungen wurde dieser Effekt jedoch nicht nachgewiesen. Wagner et al. (1996) sehen diese besseren "outcomes“ in einer effektiveren Organisation der Betreuung begründet, nicht aber im Können und Wissen des jeweiligen Arztes. Greenfield et al. (1995) konnten keine Unterschiede zwischen Spezialisten und Allgemeinärzten in den klinischen Outcomes bei nichtinsulinabhängigem Diabetes mellitus und arterieller Hypertonie entdecken (Greenfield et al. 1995). Zu vergleichbaren Ergebnissen kommt eine skandinavische Studie (Engström et al. 2001), wonach Allgemeinärzte bei gleicher Qualität sogar kostengünstiger arbeiteten als ihre spezialisierten Kollegen. Negative Einstellungen zur Allgemeinmedizin sind also nicht oder nicht nur Vorurteile, sondern auch Spiegel unterschiedlicher wissenschaftlicher Beurteilungen der Qualität hausärztlicher Versorgung.

\subsubsection{Allgemeinärztliche Entscheidungsfindung}

Auffällig in der vorliegenden Untersuchung war die große Zustimmung zum Vorwurf der Unwissenschaftlichkeit allgemeinmedizinischer Entscheidungsfindung. Die typische allgemeinmedizinische Konsultation ist durch ein komplexes multidimensionales Gefüge bestimmt, in dem oftmals eine einfache leitlinienorientierte 
Entscheidung nicht möglich ist (Abholz 2000c). Während die meisten Fächer zahlreiche Leitlinien zur Sicherung von Qualitätsstandards erarbeitet haben, gab es in der deutschen Allgemeinmedizin 2000 lediglich eine einzige Leitlinie (HummersPradier und Kochen 2000), 21 weitere waren in Arbeit (Beyer et al. 2000). Leitlinien sollen evidenzbasiert sein. Häufig existieren für den ambulanten Rahmen jedoch keine Studien oder die existierenden Studien und Leitlinien einzelner nicht allgemeinmedizinischer Fachgesellschaften sind auf die allägliche Praxiswirklichkeit nicht übertragbar (Abholz 2000c, Becker und Kochen 2001, Hummers-Pradier et al. 2000, van Weel und Knottnerus 1999). Die negative Haltung der Befragten zur Wissenschaftlichkeit hausärztlicher Medizin könnte auch in der nur selten „hautnah“ erlebten, komplexen Anforderung hausärztlicher Praxis begründet liegen. Es besteht ein großer Forschungsbedarf, um die Vielschichtigkeit allgemeinmedizinischer Arbeit adäquat zu beschreiben und entsprechende Empfehlungen zu erarbeiten. Dass Allgemeinärzte zumeist über Behandlungsstandards informiert sind und auch im Rahmen der Praktikabilität danach handeln und behandeln, zeigen zwei englische Untersuchungen (Freeman und Sweeney 2001, Gill et al 1996).

\subsubsection{Allgemeinmedizinische Forschung}

Ein wesentliches Gütekriterium, an dem sich die wissenschaftliche Allgemeinmedizin messen lassen muss, sind ihre Forschungsergebnisse (Curtis und Smith 1997, Mainous III et al. 2000, Hilton und Carter 2000). Die wissenschaftliche Allgemeinmedizin steht in Deutschland im Gegensatz zu Großbritannien, den Niederlanden und den USA noch am Anfang ihrer Entwicklung. Obwohl es in den letzten Jahren im Ausland (van Weel 1999, Levasseur et al. 2001) und Inland (Kochen et al. 2000) zu einem Aufschwung in der primärmedizinischen Forschung und einem breiten Interesse auch fachfremder Disziplinen an ihren Ergebnissen gekommen ist, wurde die allgemeinmedizinische Forschung in der vorliegenden Studie sehr negativ beurteilt. Nur 15\% der Hochschullehrer hielten die Qualität der Forschung anderen Fächern vergleichbar und nur die Hälfte der Befragten werteten die Bedeutung der Forschung als anderen Fachgebieten ebenbürtig. Allerdings versprachen sich $70 \%$ der Befragten aus der allgemeinmedizinischen Forschung Erkenntnisse, die multidisziplinär verwertbar sind (Tabelle 3). 
Wie bereits oben angeführt, ist der Forschungsbedarf in der Primärmedizin auch im Hinblick auf Veränderungen im Gesundheitssystem groß (Jones 2000, Hilton und Carter 2000, Carter und Hilton 2000, Morrison und Watt 2001, van Weel 1999, van Weel et al. 2000, Abholz 1997), die Rahmenbedingungen hierfür sind jedoch unausgereift. Für eine qualitativ hochwertige Forschung ist eine entsprechende personelle und finanzielle Ausstattung sowie unterstützende Infrastruktur nötig (Kochen et al. 2000). Untersuchungen aus dem englischsprachigen Ausland (Allen et al. 1993, Mainous III et al. 2000) verweisen auf die Schwierigkeiten der zumeist kleinen Abteilungen für Allgemeinmedizin, die „kritische Masse“ für die Anforderungen von Forschung, Lehre und Krankenversorgung zu erbringen.

Die Besonderheiten allgemeinmedizinischer Tätigkeit (ambulante Kontakte, unausgelesenes Patientengut, Langzeitbetreuung, unspezifische Symptome) spiegeln sich in den primärmedizinischen Forschungsthemen wider (van Weel 1999). Primärärztliche Forschungsnetzwerke haben sich bei Erfassung und Auswertung umfangreicher Patientendaten bewährt (van Weel et al. 2000). Die meisten Netzwerke arbeiten mehr oder weniger universitätsbasiert (Nutting 1996, Nutting et al. 1999). Britische Autoren verweisen darauf, dass in Hinblick auf die intensiver werdende primärmedizinische Forschung die Einrichtung lokaler Forschungsnetzwerke neue Möglichkeiten bietet und so eine Entlastung der universitären Abteilungen bewirken könnte (Morrison und Watt 2000). Dass Universitätsabteilungen und Fachgesellschaften bei der Entwicklung und Organisation primärmedizinischer Forschung eine wichtige Rolle spielen (werden), belegt eine deutsche Studie. Deutsche Allgemeinärzte mit Interesse an einer wissenschaftlichen Weiterqualifikation erwarten von beiden Einrichtungen formale und methodische Unterstützung bei Forschungsprojekten (Himmel und Kochen 1997).

Nur 25\% der in dieser Studie befragten Hochschullehrer glaubten, dass Allgemeinärzte an Forschung interessiert seien. Tatsächlich gestaltet sich die Rekrutierung qualifizierter Forscher und motivierter Allgemeinärzte oftmals schwierig (Allen et al. 1993, Mainous III 2000). Eine deutsche Untersuchung widerlegt aber das Vorurteil, dass Praktiker generell nicht an Forschung interessiert seien. Niedergelassene allgemeinmedizinisch tätige Ärzte äußerten sich grundsätzlich positiv zur Teilnahme an wissenschaftlichen Studien (Muche et al. 2000). Um dieses Potenzial zu nutzen, müssen entsprechende Rahmenbedingungen geschaffen werden. Ein 
Anfang wurde in Deutschland durch die vom Bundesministerium für Forschung und Bildung im Jahr 1999 ausgeschriebene Förderung der Forschung in der Allgemeinmedizin gemacht.

\subsubsection{Allgemeinmedizinische Lehre}

Traditionell hat und hatte die Lehre in der Allgemeinmedizin Priorität (Allen et al.1993, Amon et al. 1997). Während in den meisten Fachbereichen Forschung den höchsten Stellenwert hat, gaben allgemeinmedizinische Abteilungsleiter Aus- und Weiterbildung den Vorrang (Mainous III et al. 2000). Bei Block et al. (1996) wurde die allgemeinmedizinische Lehre - im Gegensatz zu allen anderen Bereichen - sehr positiv beurteilt. Erstaunlich ist daher die kritische Bewertung der Lehrqualität in unserer Erhebung (Tabelle 3). Die allgemeinmedizinische Lehre ist überwiegend an den Universitätskliniken angesiedelt und oftmals durch theoretische Stoffvermittlung ohne direkten Patienten- und Praxiskontakt charakterisiert. Mittlerweile ist die Lehre in der Allgemeinmedizin an den meisten Universitäten im Umbruch. Durch die Einführung von Pflichtpraktika und Hospitationen (z.B. Abteilung Allgemeinmedizin der Universität Göttingen; Sektion Allgemeinmedizin der Universität Heidelberg) wird ein Einblick in die Primärmedizin möglich, den die meisten Studenten als Bereicherung erleben (Amon et al. 1997, Himmel 2000, Sohn 1997). Auch die überwiegende Mehrheit der Patienten empfindet die Anwesenheit eines Studenten in Lehrpraxen nicht als störend, sondern als Auszeichnung (Bentham et al. 1999, Simons et al. 1995, Stunder 2000). In einer britischen Studie waren fast alle Patienten bereit, an Unterrichtsprogrammen teilzunehmen (Jones und Oswald 1994). Für Studenten scheint der Erwerb von grundlegenden klinischen Fähigkeiten im ambulanten Rahmen ebenso gut möglich zu sein wie in der Universitätsklinik. Sie schätzten vor allem die Möglichkeit, nicht-selektierte Patienten zu sehen (Parle et al. 1997). Eine Verbesserung der Lehrqualität in der Allgemeinmedizin dürfte zur Profilbildung des Faches in den medizinischen Fakultäten beitragen (Siebolds et al. 2000).

\subsubsection{Stärkung der Allgemeinmedizin im Studium - Einflussfaktoren}

Wie wichtig die wissenschaftliche Allgemeinmedizin in der Fremdeinschätzung ist, verdeutlicht der Einfluss, den sie auf den Wunsch der Befragten hatte, die Allgemeinmedizin im Studium zu stärken. Abteilungsdirektoren und Dekane, die eine positive 
Haltung zur akademischen Allgemeinmedizin hatten, waren nach eigener Aussage häufiger bereit, die allgemeinmedizinische Position im Curriculum zu stärken. Einen ähnlichen Effekt hatte die positive Einschätzung primärmedizinischer Versorgung (Tabelle 11). Diese Zusammenhänge legen es nahe, durch qualitativ hochstehende Beiträge an der Profilbildung des akademischen Faches Allgemeinmedizin weiterzuarbeiten.

In zahlreichen Studien zur Berufswahl „Allgemeinmedizin“ erwiesen sich die Präsenz einer starken universitären Abteilung für Allgemeinmedizin und ein langjähriger Kontakt mit der Allgemeinmedizin als relevante Prädiktoren (Bland et al. 1995, Wilm 1997, Schafer et al. 2000, Senf et al. 1997). In unserer Studie dagegen schienen diese Faktoren ${ }^{3}$ ebenso wie strukturelle Aspekte (Alter, Bundesland) die Meinungsbildung von Hochschullehrern nicht signifikant zu beeinflussen (Tabelle 10).

\subsubsection{Allgemeinmedizin und Innere Medizin}

Der Wunsch, die Allgemeinmedizin im Studium zu stärken, wurde signifikant durch die Abteilungszugehörigkeit beeinflusst. Bereits in der bivariaten Analyse nach Klinikzugehörigkeit fiel eine deutliche Polarisierung des Antwortverhaltens auf: Befragte aus den Fächern Psychiatrie/Psychosomatik und die Dekane vertraten eine deutlich positivere Haltung zur Allgemeinmedizin als ihre Kollegen aus der Inneren Medizin (Tabelle 7). Diese Tendenz bestätigte sich auch im multivariaten Modell (Tabelle 10 und 11). Danach hatten weitaus mehr Professoren aus Psychiatrie/ Psychosomatik als Direktoren der Inneren Medizin Interesse an einer Aufwertung der Allgemeinmedizin im Studium. Möglicherweise kommt hier die gemeinsame sozialwissenschaftliche Ausrichtung der erstgenannten Fächer zum Tragen. Z. B. hielten drei Viertel der Direktoren aus der Psychiatrie eine verstärkte Integration sozialwissenschaftlicher Methoden ins Studium für wünschenswert, bei den Internisten waren es $37 \%$.

\footnotetext{
${ }^{3}$ Im Einzelnen waren dies: Bekanntschaft mit dem Leiter der Allgemeinmedizin, gemeinsame Projekte, positive Erfahrung mit der universitären Allgemeinmedizin als Indikatoren für eine „starke Allgemeinmedizin“ und eigene Arbeitserfahrung in der Allgemeinmedizin
} 
Innere Medizin und Allgemeinmedizin stehen sich inhaltlich und in Hinblick auf die Aus- und Weiterbildung ${ }^{4}$ relativ nahe. Oftmals ist die Abteilung für Allgemeinmedizin in ein übergeordnetes Zentrum für Innere Medizin integriert (so z.B. an der Universität Göttingen). Hat die Innere Medizin für sich jahrelang in Anspruch nehmen können, den „allgemeinsten“ Bereich der klinischen Ausbildung zu vermitteln (Heimpel 1997), so wird die Etablierung der universitären Allgemeinmedizin möglicherweise negativ als Konkurrenz erlebt. Diese Spannungssituation findet ihre Fortsetzung in der aktuellen gesundheitspolitischen Debatte um die hausärztliche Versorgung durch Allgemeinarzt und/oder Internist (Maus 2001a, Maus 2001b, Korzilius 2001a, Korzilius 2001b, Richter 2001b). Die Etablierung eines gemeinsamen Weiterbildungszweiges „Hausarztmedizin“ von Allgemeinmedizin und allgemeiner Innerer Medizin könnte auch in Hinblick auf den zukünftigen Bedarf an Hausärzten neue Perspektiven bieten (Donner-Banzhoff 2000, Maus 2001c).

Das Verhältnis von Innerer Medizin und Primärmedizin beleuchtet auch die amerikanische Studie von Zinn et al. (1998). Primärmedizinische Versorgung wird in den USA gleichermaßen durch die Fächer „Family Medicine“, „General Internal Medicine“ und "General Pediatrics“ vertreten. Aufgrund dieser Struktur sind die Ergebnisse und Schlussfolgerungen auf unser Gesundheitssystem nicht übertragbar, illustrieren aber einige interessante Aspekte: Abteilungsangehörige der „Family Medicine“/Allgemeinmedizin waren gegenüber primärmedizinischen Belangen positiver eingestellt und öfter bereit, eine Förderung der Allgemeinmedizin im Studium zu unterstützen, als ihre Kollegen aus der allgemeinen Inneren Medizin („General Internal Medicine“). Die Autoren vermuteten einen Zusammenhang zwischen der universitären Etablierung ${ }^{5}$ und der unterschiedlichen medizinischen Kultur in beiden Fächern und diesen Ergebnissen (Schwenk 1998, Zinn et al. 1998).

\footnotetext{
${ }^{4}$ Weiterbildungsordnung Allgemeinmedizin: 1,5 Jahre Allgemeinmedizin, 1 Jahr Innere Medizin im Stationsdienst, 0,5 Jahre Chirurgie, 0,5 Jahre Pädiatrie, 1,5 Jahre „freie“ Weiterbildung (Weiterbildungsordnung Allgemeinmedizin für Niedersachsen vom 1. Mai 1999)

5 „Family Medicine“ ist als eigene Disziplin institutionalisiert. „General Internal Medicine“ gehört als Subdisziplin zur Inneren Medizin.
} 


\subsubsection{Entwicklungen in der Allgemeinmedizin - USA und Europa}

In der internationalen Entwicklung der Allgemeinmedizin gibt es zur Zeit zwei gegensätzliche Tendenzen. In den USA zeichnet sich eine Stagnation ab, die ihren Ausdruck im Rückgang der Anzahl der Bewerber für Weiterbildungsprogramme in Allgemeinmedizin findet. Anfang der 90er Jahre fehlten vor allem in den ländlichen Gebieten der Vereinigten Staaten zahlreiche Allgemeinärzte. Die daraufhin initiierten Programme zur Steigerung der Zahl der Absolventen, die eine Weiterbildung in Allgemeinmedizin anstrebten, waren zunächst erfolgreich (Blake 1999). Seit 1997 ist die Zahl der „Family Medicine Residents“ allerdings kontinuierlich zurückgegangen (Pugno et al. 2000, Pugno et al. 2001). Die AAFP ${ }^{6}$ berichtete anlässlich des "Weiterbildungszulassungsverfahrens" 2001, dass die meisten jungen Allgemeinärzte aus den Universitäten der ländlich geprägten Staaten des Mittleren Westen kamen, wo ein hoher primärmedizinischer Versorgungsbedarf besteht. In diesen Universitäten sind Abteilungen für Allgmeinmedizin etabliert, die z.T. entsprechende Ausbildungsschwerpunkte (z.B. „rural medicine") anbieten (University of Minnesota 2002). Am unteren Ende der Rangliste fanden sich die großen renommierten biomedizinisch ausgerichteten Forschungsuniversitäten (Columbia University, Yale University, Harvard Medical School). Diese sind zumeist in den urbanen Ostküstenstaaten angesiedelt und haben keine Abteilungen für Allgemeinmedizin etabliert. Die Entwicklung und Stärke der universitären Allgemeinmedizin scheint also wesentlich von gesundheitspolitischen Prioritäten und Entscheidungen, aber auch von gesundheitsgeographischen Erfordernissen bestimmt zu sein. Die fehlende Institutionalisierung an den Eliteuniversitäten lässt die Allgemeinmedizin als Forschungsfach zweiter Klasse erscheinen und stellt die Frage nach Anspruch und Wirklichkeit akademischer Allgemeinmedizin in den USA.

In Europa erlebt die Allgemeinmedizin einen Aufschwung. Fortschritte in Lehre und Forschung und auch die fortschreitende universitäre Etablierung des Faches (Kochen et al. 2000, van Weel 1999, Brinkley 2001, Levasseur et al. 2001, Svab et al. 2001) gehen einher mit einem gestiegenen Interesse an diesen Ergebnissen. Insgesamt herrschen gute Bedingungen für eine positive Entwicklung der

\footnotetext{
${ }^{6}$ AAFP $=$ American Association of Family Practitioners
} 
akademischen Allgemeinmedizin, zumal sich die Allgemeinmedizin sowohl gesundheits- als auch bildungspolitisch zu einem wichtigen Partner entwickelt.

\subsection{Schlussfolgerungen}

Bereits in den 80 er Jahren zeigte eine Studie bei 1.200 niedergelassenen Fach- und Krankenhausärzten eine überwiegend positive Einschätzung der Rolle des Allgemeinarztes im Gesundheitssystem und eine Akzeptanz der Eigenständigkeit des Faches Allgemeinmedizin. Als wichtige Aufgabe des Allgemeinarztes wurde u.a. die Betreuung chronisch und unheilbar Kranker gesehen. Die Rolle des Allgemeinarztes könne nicht ohne weiteres von einem Spezialisten ausgeübt werden, meinten $46 \%$ der damals Befragten. ${ }^{7}$

Bezogen sich diese damaligen Einschätzungen vorwiegend auf die hausärztliche Patientenversorgung, konnte die vorliegende Untersuchung auch eine überwiegend positive Haltung von medizinischen Hochschullehrern zur universitären Allgemeinmedizin zeigen. In den letzten Jahren ist die Allgemeinmedizin fester Bestandteil der medizinischen Ausbildung geworden und eine Stärkung ihrer Position wurde von den Befragten mehrheitlich befürwortet. Um sich im akademischen Miteinander als vollwertiger Partner zu etablieren, wird die Allgemeinmedizin noch stärker als bisher eine qualitativ hochwertige wissenschaftliche Basis in Forschung, Lehre und Krankenversorgung entwickeln und aufbauen müssen. Grundlage für diese Entwicklung ist eine kontinuierliche Institutionalisierung des Faches mit einer entsprechenden finanziellen und personellen Ausstattung an den deutschen medizinischen Hochschulen. In Anbetracht der positiven Haltung der befragten Abteilungsdirektoren und Dekane kann die universitäre Allgemeinmedizin positiven Rückhalt in den Fakultäten erwarten.

\footnotetext{
${ }^{7}$ Leider ist diese durch das Institut Infratest durchgeführte Umfrage heute nicht mehr einzusehen (schriftliche Auskunft Infratest 2001) und muss daher aus zweiter Hand zitiert werden (Sturm 1983).
} 


\section{$7 \quad$ Zusammenfassung}

Hintergrund: Reformen im Medizinstudium und Gesundheitswesen fordern eine Aufwertung der Allgemeinmedizin. Trotz langjähriger Diskussionen ist das Fach an den meisten Universitäten noch immer unzureichend etabliert.

Fragestellung: Wie bewerten innerfakultäre Entscheidungsträger an den deutschen medizinischen Hochschulen die akademische Allgemeinmedizin?

Methode: Standardisierte schriftliche Befragung aller Dekane und Abteilungsdirektoren für Innere Medizin, Chirurgie, Pädiatrie, Gynäkologie und Psychiatrie/Psychosomatik an den 37 deutschen medizinischen Fakultäten.

Ergebnisse: 79\% (474/596) der angeschriebenen Hochschullehrer nahmen an der Befragung teil. Die überwiegende Mehrheit (87\%) bescheinigte der Allgemeinmedizin eine wichtige Aufgabe im deutschen Gesundheitswesen. Zwei Drittel der Befragten hielten Spezialisten für weniger geeignet als Allgemeinärzte, die primärärztliche Versorgung sicherzustellen und $50 \%$ hielten den Allgemeinarzt für besonders geeignet, Patienten mit chronischen und fortschreitenden Erkrankungen zu betreuen. Während fast die Hälfte der Teilnehmer (223/464) die Bedeutung allgemeinmedizinischer Forschung als anderen Fächern gleichwertig einschätzte, waren es für die Qualität allgemeinmedizinischer Forschung nur 14\%. Auch die Qualität allgemeinmedizinischer Lehre bewertete lediglich jeder Dritte (138/468) als anderen Fächern vergleichbar. Zwei Drittel der Befragten (314/468) befürworteten eine Stärkung der Allgemeinmedizin im Studium. In der multivariaten Analyse waren an einer Stärkung der Allgemeinmedizin besonders Professoren interessiert, die eine positive Einstellung zur Primärmedizin hatten (Odds ratio [OR] 2,6; 95\%-Konfidenzintervall [KI] 1,6-4,3) und die wissenschaftliche Allgemeinmedizin vergleichsweise positiv bewerteten (OR 5,5; 95\%-KI 2,9-10,4). Im Vergleich zu ihren Kollegen aus Psychiatrie/ Psychosomatik waren die Professoren aus der Inneren Medizin seltener an einer Stärkung interessiert (OR 0,48; 95\%-KI 0,3-0,8).

Schlussfolgerungen: Obwohl bei Dekanen und Abteilungsdirektoren eine positive Grundstimmung dominierte, muss die akademische Allgemeinmedizin auch in Zukunft durch die Qualität ihrer Beiträge zu Forschung und Lehre zu ihrer Akzeptanz an den Universitäten beitragen. 


\section{$8 \quad$ Anhang}

\subsection{Teilnehmende medizinische Fakultäten}

Aachen: Rheinisch-Westfälische Technische Hochschule

Berlin: Freie Universität

Berlin: Humboldt-Universität

Bochum: Ruhr-Universität

Bonn: Rheinische Friedrich-Wilhelms-Universität

Dresden: Technische Universität

Düsseldorf: Heinrich-Heine-Universität

Erlangen-Nürnberg: Friedrich-Alexander-Universität

Essen: Universität Essen

Frankfurt/Main: Johann Wolfgang Goethe-Universität

Freiburg: Albert-Ludwigs-Universität

Gießen: Justus-Liebig-Universität

Göttingen: Georg-August-Universität

Greifswald: Ernst-Moritz-Arndt-Universität

Halle-Wittenberg: Martin-Luther-Universität

Hannover: Medizinische Hochschule

Heidelberg: Ruprecht-Karls-Universität

Hamburg: Universität Hamburg

Homburg/Saar: Universität des Saarlandes

Jena: Friedrich-Schiller-Universität

Kiel: Christian-Albrechts-Universität

Köln: Universität zu Köln

Leipzig: Universität Leipzig

Lübeck: Medizinische Universität

Magdeburg: Otto-von-Guericke-Universität

Mainz: Johannes Gutenberg-Universität

Mannheim: Fakultät für Klinische Medizin Mannheim der Universität Heidelberg

Marburg: Philipps-Universität

München: Ludwig-Maximilians-Universität 
München: Technische Universität

Münster: Westfälische Wilhelms-Universität

Regensburg: Universität Regensburg

Rostock: Universität Rostock

Tübingen: Eberhard-Karls-Universität

Ulm: Universität UIm

Witten/Herdecke: Universität Witten/Herdecke

Würzburg: Julius-Maximilians-Universität 


\subsection{Fragebogen}

Bitte kreuzen Sie auf den folgenden Seiten jeweils die Antwort an, die Ihrer persönlichen Meinung und Erfahrung am ehesten entspricht. Nur an einigen Stellen bitten wir Sie um eine kurze schriftliche Ergänzung. Die Bearbeitung des Fragebogens wird Sie knapp 10 Minuten in Anspruch nehmen.

\section{$\Rightarrow$ Zunächst einige Aussagen zur Allgemeinmedizin}

1. Allgemeinärzte müssen mit einer größeren diagnostischen und therapeutischen Unsicherheit arbeiten als Spezialisten.

2. Spezialisten können genauso gut wie Allgemeinärzte die primärärztliche Versorgung sicherstellen.

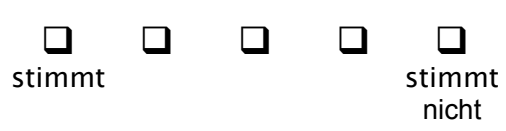

3. Allgemeinärzte haben im deutschen Gesundheitssystem eine wichtige Aufgabe.

4. Allgemeinärzte sind besonders befähigt, Patienten bei chronischen und fortschreitenden Krankheiten zu betreuen.

5. Allgemeinärzte sind in ihren Entscheidungen stärker durch Wünsche des Patienten beeinflußt als Klinikärzte. $\underset{\text { stimmt }}{\square} \quad \square \quad \square \quad \square \quad \underset{\substack{\text { stimmt } \\ \text { nicht }}}{\square}$<smiles>[13CH]=[13CH][Hg]</smiles>

6. Die Methoden der Entscheidungsfindung in der

Allgemeinmedizin sind weniger wissenschaftlich als die Methoden in den Spezialdisziplinen.

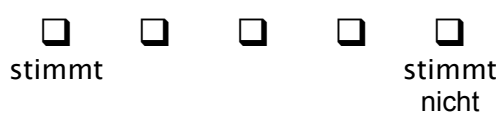

7. Allgemeinmediziner sind "Schmalspurmediziner".

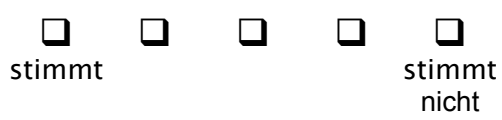

\section{$\Rightarrow$ Wie bewerten Sie folgende Aussagen zur allgemeinmedizinischen Forschung und Lehre?}

8. Wie bewerten Sie die Wichtigkeit allgemeinmedizinischer Forschung im Vergleich zu anderen medizinischen Fächern?

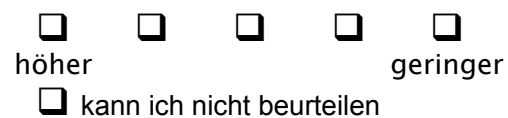


9. Wie bewerten Sie die Qualität allgemeinmedizinischer Forschung im Vergleich zu anderen medizinischen Fächern?

10. Sozialwissenschaftliche Methoden sollten in der medizinischen Forschung und Lehre gleichberechtigt neben naturwissenschaftlichen Methoden stehen.

11. Allgemeinärzte haben wenig Interesse, an wissenschaftlicher Forschung teilzunehmen.

12. Forschung in der Allgemeinmedizin bringt auch für andere medizinische Fachgebiete wichtige Erkenntnisse.

13. Wie bewerten Sie die Qualität allgemeinmedizinischer Lehre im Vergleich zu anderen medizinischen Fächern?

14. Wie bewerten Sie die Wichtigkeit allgemeinmedizinischer Lehre im Vergleich zu anderen medizinischen Fächern?

15. Die Lehre in der Allgemeinmedizin unterscheidet sich von anderen medizinisch-klinischen Fächern durch Vermittlung von Handlungsstrategien statt reiner Wissensvermittlung.

16. Die Allgemeinmedizin erfordert Wissen, das durch die einzelnen Spezialdisziplinen nicht vermittelt werden kann.

17. Wird Ihrer Meinung nach dieses Wissen im universitären Medizinstudium ausreichend vermittelt?
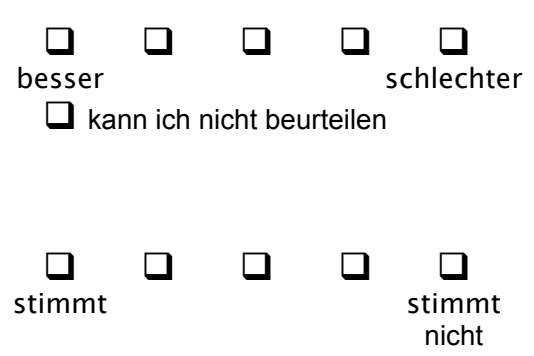

$\underset{\text { stimmt }}{\square} \square \quad \square \quad \square \quad \underset{\substack{\text { stimmt } \\ \text { nicht }}}{\square}$
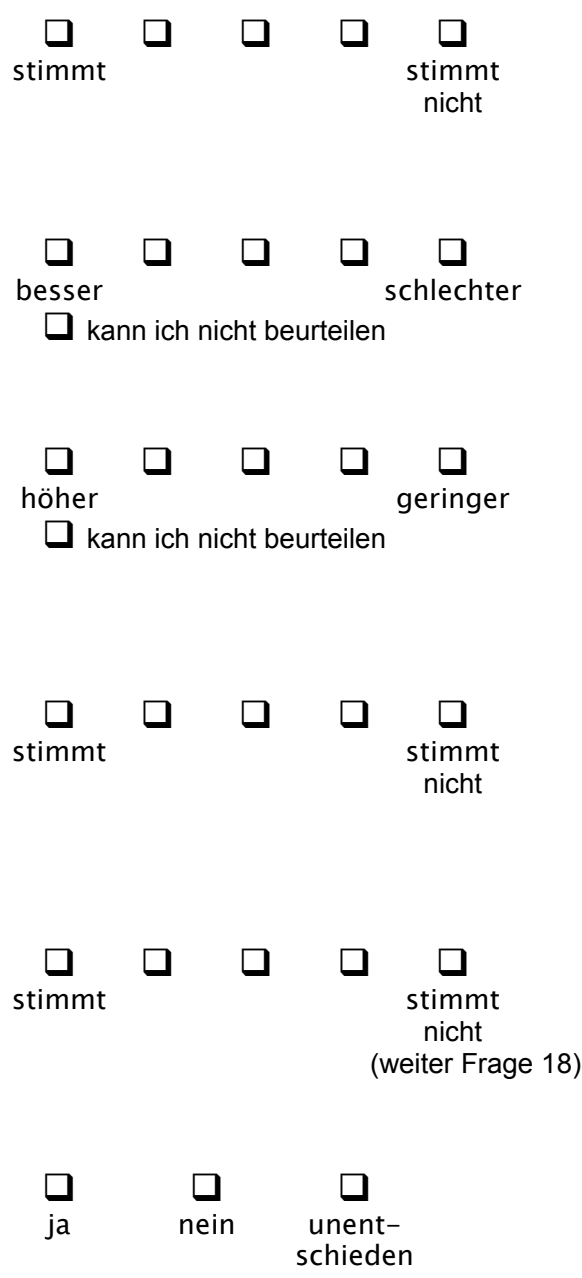
$\Rightarrow$ Wie wichtig werden Ihrer Meinung nach folgende Inhalte für die zukünftige Medizinerausbildung sein?

18. Geriatrie

19. Therapie chronischer Krankheiten

20. Multimorbidität

21. Familienmedizin

22. ambulante Versorgung

23. rationale Arzneimitteltherapie

24. Kenntnisse des Sozialsystems

25. Gesundheitsförderung

26. Gesprächsführung

27. PC-Einsatz für Literaturrecherche

28. PC-Einsatz für Wissensvermittlung

29. PC-Einsatz für Dokumentation und Abrechnung

30. ökonomische Konsequenzen ärztlichen Handelns

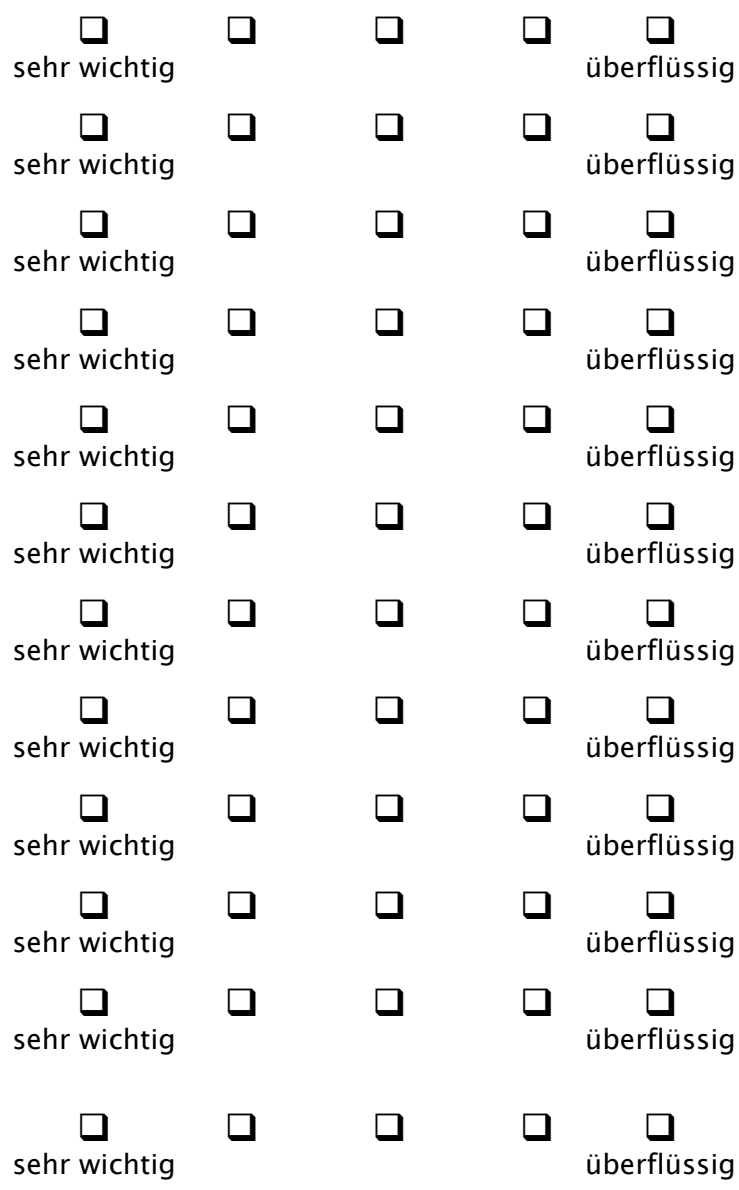

$\underset{\text { sehr wichtig }}{\square}$

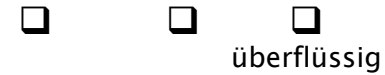

\section{$\Rightarrow$ Die Allgemeinmedizin an Ihrer Universität}

31. Wie ist die Allgemeinmedizin an Ihrer Universität repräsentiert?

32. Sind Sie der Meinung, daß dieses Modell beibehalten werden soll?

33. Kennen Sie den leitenden Fachvertreter der Allgemeinmedizin an Ihrer Universität persönlich?

\footnotetext{
$\square$ eigener Lehrstuhl

$\square$ Institut, Lehr-, Arbeitsbereich, o.ä.

口 Lehrbeauftragte

$\square$ andere

$\square \quad$ ist mir nicht bekannt (weiter Frage 33)
}

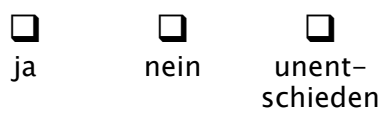

$\underset{\text { ja }}{\square} \underset{\text { nein }}{\square}$ 
34. Gibt es gemeinsame Projekte mit der Allgemeinmedizin an Ihrer Universität?

35. Hat Ihre Abteilung Kontakte zu den Allgemeinärzten Ihrer Region, z.B. "Runder Tisch"?

36. Welche Erfahrungen haben Sie im Laufe Ihrer ärztlichen Tätigkeit mit der universitären Allgemeinmedizin gemacht?

37. Welche Erfahrungen haben Sie im Laufe Ihrer ärztlichen Tätigkeit mit der praktischen Allgemeinmedizin gemacht?

38. Würden Sie eine Stärkung der Allgemeinmedizin im Rahmen des Medizinstudiums unterstützen?

39. Halten Sie die Allgemeinmedizin im Rahmen des Medizinstudiums für verzichtbar?

40. Würden Sie einen sehr guten Studenten mit dem Berufsziel „Arzt f. Allgemeinmedizin“ in dieser Wahl unterstützen?

41. Haben Sie im Rahmen Ihrer ärztlichen

Tätigkeit in der Allgemeinmedizin gearbeitet?

42. Ihre Abteilung

43. Ihr Geschlecht

44. Ihr Alter

45. Bundesland

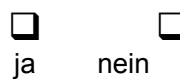

Wenn ja, bitte Projekte in Stichworten nennen:

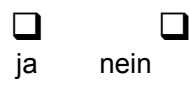

Wenn ja, bitte Projekte in Stichworten nennen:
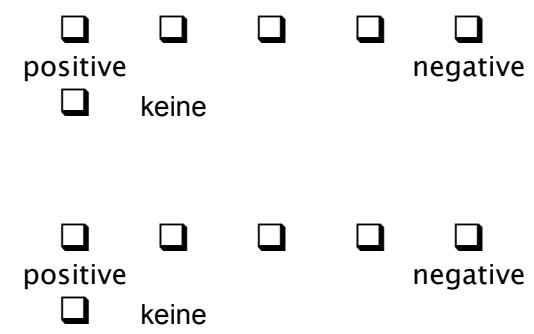

$$
\underset{\text { ja }}{\square} \underset{\begin{array}{c}
\text { nein } \\
\text { schient- }
\end{array}}{\square}
$$

$\square$ ja $\quad \underset{\text { nein }}{\square} \quad \begin{gathered}\square \\ \text { unent- } \\ \text { schieden }\end{gathered}$

$\underset{\text { ja }}{\square} \quad \underset{\text { nein }}{\square} \quad \begin{gathered}\square \\ \text { unent- } \\ \text { schieden }\end{gathered}$

$\square_{\text {ja }}^{\square} \underset{\text { nein }}{\square}$

Wenn ja, wie lange:

\section{$\square \quad$ Innere Medizin $\square$ Pädiatrie \\ $\square \quad$ Psychiatrie/Psychosomatik}

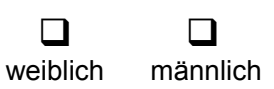




\subsection{Gesamtauswertung}

(k.A. = keine Angabe)

1. Allgemeinärzte müssen mit einer

$\mathrm{n}=470$ größeren diagnostischen und therapeutischen Unsicherheit arbeiten als $n(k . A)=$.

stimmt Spezialisten.

2. Spezialisten können genauso gut wie

$n=470$ Allgemeinärzte die primärärztliche Versorgung sicherstellen.

$\mathrm{n}(\mathrm{k} . \mathrm{A})=$.

3. Allgemeinärzte haben im deutschen

$n=469$ Gesundheitssystem eine wichtige Aufgabe.

$\mathrm{n}(\mathrm{k} . \mathrm{A})=$.

4. Allgemeinärzte sind besonders

$n=470$ befähigt, Patienten bei chronischen und fortschreitenden Krankheiten zu $n(k . A)=$. betreuen.

5. Allgemeinärzte sind in ihren

$\mathrm{n}=468$

Entscheidungen stärker durch Wünsche des Patienten beeinflußt als Klinikärzte.

6. Die Methoden der Entscheidungsfindung in der Allgemeinmedizin sind weniger wissenschaftlich als die Methoden in den Spezialdisziplinen.

7. Allgemeinmediziner sind

$n=466$

$\begin{array}{cccccc} & \square & \square & \square & \square & \square \\ \mathrm{n} & 40 & 38 & 91 & 129 & 172 \\ \% & 8,5 & 8,1 & 19,4 & 27,4 & 36,6\end{array}$

"Schmalspurmediziner".

$\mathrm{n}(\mathrm{k} . \mathrm{A})=$.

8. Wie bewerten Sie die Wichtigkeit

$\mathrm{n}=464$ allgemeinmedizinischer Forschung im Vergleich zu anderen medizinischen $\mathrm{n}(\mathrm{k} . \mathrm{A})=$. $\begin{array}{cccccc} & \square & \square & \square & \square & \square \\ \mathrm{n} & 116 & 145 & 87 & 65 & 56 \\ \% & 24,7 & 30,9 & 18,6 & 13,9 & 11,9\end{array}$ $\mathrm{n}(\mathrm{k} . \mathrm{A})=$. Fächern?
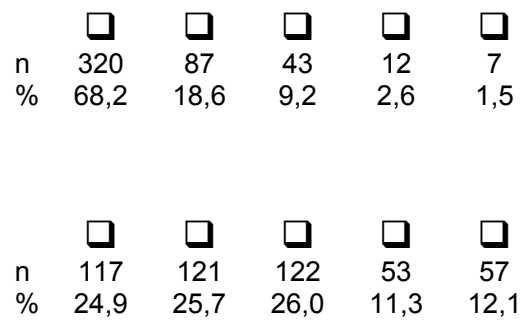
$\begin{array}{cccccc} & \square & \square & \square & \square & \square \\ \mathrm{n} & 154 & 158 & 75 & 42 & 39 \\ \% & 32,9 & 33,8 & 16,0 & 9,0 & 8,3\end{array}$ 
9. Wie bewerten Sie die Qualität allgemeinmedizinischer Forschung im Vergleich zu anderen medizinischen Fächern?

10. Sozialwissenschaftliche Methoden sollten in der medizinischen Forschung u. Lehre gleichberechtigt neben naturwissenschaftlichen Methoden stehen.

11. Allgemeinärzte haben wenig Interesse, an wissenschaftlicher Forschung teilzunehmen.

12. Forschung in der Allgemeinmedizin bringt auch für andere medizinische Fachgebiete wichtige Erkenntnisse.

13. Wie bewerten Sie die Qualität allgemeinmedizinischer Lehre im Vergleich zu anderen medizinischen Fächern?

14. Wie bewerten Sie die Wichtigkeit allgemeinmedizinischer Lehre im Vergleich zu anderen medizinischen Fächern?

15. Die Lehre in der Allgemeinmedizin unterscheidet sich von anderen medizinisch-klinischen Fächern durch Vermittlung von Handlungsstrategien statt reiner Wissensvermittlung.

16. Die Allgemeinmedizin erfordert Wissen, das durch die einzelnen Spezialdisziplinen nicht vermittelt werden kann.

17. Wird Ihrer Meinung nach dieses Wissen im universitären Medizinstudium ausreichend vermittelt? $\mathrm{n}=389$

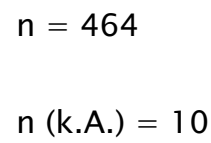

stimmt

stimmt nicht

$\begin{array}{cccccc} & \square & \square & \square & \square & \square \\ \mathrm{n} & 174 & 143 & 81 & 48 & 17 \\ \% & 37,6 & 30,9 & 17,5 & 10,4 & 3,7\end{array}$

$(k \cdot A)=85$ 
Wie wichtig werden Ihrer Meinung nach folgende Inhalte für die zukünftige Medizinerausbildung sein?

18. Geriatrie

$n=466$

$\mathrm{n}(\mathrm{k} . \mathrm{A})=$.
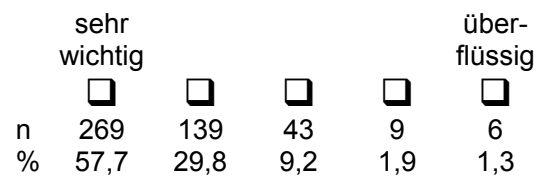

19. Therapie chronischer Krankheiten

$n=470$ $\begin{array}{cccc} & \square & \square & \square \\ \mathrm{n} & 301 & 143 & 24 \\ \% & 64 & 30,4 & 5,1\end{array}$

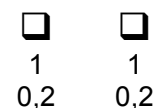

$\mathrm{n}(\mathrm{k} . \mathrm{A})=$.

20. Multimorbidität

$n=468$ $\begin{array}{cccccc} & \square & \square & \square & \square & \square \\ \mathrm{n} & 253 & 151 & 55 & 7 & 2 \\ \% & 54,1 & 32,3 & 11,8 & 1,5 & 0,4\end{array}$

n (k.A.) $=6$

21. Familienmedizin

$n=461$ $\begin{array}{cccccc} & \square & \square & \square & \square & \square \\ \mathrm{n} & 141 & 135 & 139 & 36 & 10 \\ \% & 30,6 & 29,3 & 30,2 & 7,8 & 2,2\end{array}$

$n(k . A)=$.

22. ambulante Versorgung

$n=465$ $\begin{array}{cccccc} & \square & \square & \square & \square & \square \\ \mathrm{n} & 216 & 177 & 64 & 5 & 3 \\ \% & 46,5 & 38,1 & 13,8 & 1,1 & 0,6\end{array}$

$\mathrm{n}(\mathrm{k} . \mathrm{A})=$.

23. rationale Arzneimitteltherapie

$n=468$ $\begin{array}{cccccc} & \square & \square & \square & \square & \square \\ \mathrm{n} & 305 & 125 & 31 & 6 & 1 \\ \% & 65,2 & 26,7 & 6,6 & 1,3 & 0,2\end{array}$

$\mathrm{n}(\mathrm{k} . \mathrm{A})=$.

24. Kenntnisse des Sozialsystems

$n=471$ $\begin{array}{cccccc} & \square & \square & \square & \square & \square \\ \mathrm{n} & 164 & 186 & 105 & 16 & 0 \\ \% & 34,8 & 39,5 & 22,3 & 3,4 & 0\end{array}$ $\mathrm{n}(\mathrm{k} . \mathrm{A})=$.

25. Gesundheitsförderung

$n=467$ $\begin{array}{cccccc} & \square & \square & \square & \square & \square \\ \mathrm{n} & 207 & 162 & 82 & 12 & 4 \\ \% & 44,3 & 34,7 & 17,6 & 2,6 & 0,9\end{array}$ $n(k . A)=$.

26. Gesprächsführung

$n=468$ $\begin{array}{cccccc} & \square & \square & \square & \square & \square \\ \mathrm{n} & 260 & 136 & 60 & 10 & 2 \\ \% & 55,6 & 29,1 & 12,8 & 2,1 & 0,4\end{array}$ $n(k . A)=$.

27. PC-Einsatz für Literaturrecherche

$\mathrm{n}=468$ $\begin{array}{cccccc} & \square & \square & \square & \square & \square \\ \mathrm{n} & 161 & 149 & 117 & 34 & 7 \\ \% & 34,4 & 31,8 & 25,0 & 7,3 & 1,5\end{array}$ $n(k . A)=$. 
28. PC-Einsatz für Wissensvermittlung

$\mathrm{n}=468$ $\begin{array}{cccccc} & \square & \square & \square & \square & \square \\ \mathrm{n} & 149 & 160 & 126 & 30 & 3 \\ \% & 31,8 & 34,2 & 26,9 & 6,4 & 0,6\end{array}$

$$
\mathrm{n}(\mathrm{k} . \mathrm{A} .)=6
$$

29. PC-Einsatz für Dokumentation und

$\mathrm{n}=468$ Abrechnung

$$
n(k . A .)=6
$$

30. ökonomische Konsequenzen

$\mathrm{n}=468$

$\begin{array}{lrr} & \square & \square \\ \mathrm{n} & 215 & 149 \\ \% & 45,9 & 31,8\end{array}$

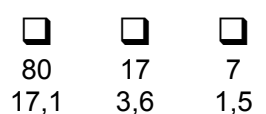
ärztlichen Handelns $\begin{array}{cccccc} & \square & \square & \square & \square & \square \\ \mathrm{n} & 243 & 154 & 64 & 5 & 2 \\ \% & 51,9 & 32,9 & 13,7 & 1,1 & 0,4\end{array}$

$$
\mathrm{n}(\mathrm{k} . \mathrm{A} .)=6
$$

31. Wie ist die Allgemeinmedizin an Ihrer $n=468$ Universität repräsentiert?

$$
n(k . A .)=8
$$

$\begin{array}{rrl}\text { N } & \% & \\ 123 & 26,4 & \square \text { Lehrstuhl } \\ 63 & 13,5 & \square \text { Lehrbereich } \\ 215 & 46,1 & \square \text { Lehrauftrag } \\ 15 & 3,2 & \square \text { andere } \\ 50 & 10,7 & \square \text { unbekannt }\end{array}$

32. Sind Sie der Meinung, daß dieses

$\mathrm{n}=417$

Modell beibehalten werden soll?

$n($ k.A. $)=57$

$\begin{array}{cccc} & \text { ja } & \text { nein } & \begin{array}{c}\text { unent- } \\ \text { schieden }\end{array} \\ & \square & \square & \square \\ \mathrm{n} & 248 & 97 & 72 \\ \% & 59,5 & 23,3 & 17,3\end{array}$

33. Kennen Sie den leitenden

$\mathrm{n}=455$

Fachvertreter der Allgemeinmedizin an Ihrer Universität persönlich?

$\mathrm{n}(\mathrm{k} . \mathrm{A})=$.

$\begin{array}{ccc} & \text { ja } & \text { nein } \\ & \square & \square \\ \mathrm{n} & 275 & 180 \\ \% & 60,4 & 39,6\end{array}$

34. Gibt es gemeinsame Projekte mit der

$n=444$ Allgemeinmedizin an Ihrer Universität? $\mathrm{n}(\mathrm{k} . \mathrm{A})=$.

$\begin{array}{ccc} & \text { ja } & \text { nein } \\ & \square & \square \\ \mathrm{n} & 115 & 325 \\ \% & 26,2 & 73,8\end{array}$

Wenn ja, welche?

(Mehrfachnennung möglich)

$\begin{array}{lr}\text { Qualitätssicherung } & 6 \\ \text { Forschung } & 10 \\ \text { Lehre } & 18 \\ \text { Klinische Projekte } & 26 \\ \text { Fort/Weiterbildung } & 3 \\ \text { Andere } & 12 \\ \text { k.A. } & 49\end{array}$


35. Hat Ihre Abteilung Kontakte zu den

Allgemeinärzten Ihrer Region, z.B.

"Runder Tisch"?

Wenn ja, welche?

(Mehrfachnennung möglich)

36. Welche Erfahrungen haben Sie im Laufe Ihrer ärztlichen Tätigkeit mit der universitären Allgemeinmedizin gemacht?

37. Welche Erfahrungen haben Sie im Laufe Ihrer ärztlichen Tätigkeit mit der praktischen Allgemeinmedizin gemacht?

38. Würden Sie eine Stärkung der Allgemeinmedizin im Rahmen des Medizinstudiums unterstützen?

39. Halten Sie die Allgemeinmedizin im Rahmen des Medizinstudiums für verzichtbar?

40. Würden Sie einen sehr guten Studenten mit dem Berufsziel „Arzt für Allgemeinmedizin“ in dieser Wahl unterstützen? $n=465$

$n(k . A)=$.

$\begin{array}{ccc} & \text { ja } & \text { nein } \\ & \square & \square \\ \mathrm{n} & 174 & 291 \\ \% & 37,4 & 62,6\end{array}$

Qualitätssicherung Forschung

Lehre

Klinische Projekte

Fort/Weiterbildung

Andere

k.A.

$n=468$

$\mathrm{n}(\mathrm{k} . \mathrm{A})=$.

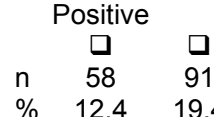

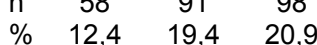

$\begin{array}{cc} & \text { negative } \\ \square & \square \\ 23 & 16 \\ 4,9 & 3,4\end{array}$

$\square$ keine

n 182

$\% \quad 38,9$

$n=468$

Positive

$\begin{array}{ccc} & \square & \square \\ \mathrm{n} & 106 & 143 \\ \% & 22,7 & 30,6\end{array}$

$\mathrm{n}(\mathrm{k} . \mathrm{A})=$.

$\square$ keine

n 50

$\% \quad 10,7$

$n=468$

$n(k . A)=$.

ja

n $\quad \square$

14

nein

unentschieden

$\square$

$\square$

$\% \quad 67,1$

12,6

20,3

$n=470$

$n(k . A)=$.

$\begin{array}{ll} & \square \\ \mathrm{n} & 42 \\ \% & 8,9\end{array}$

nein

unentschieden

$\square$

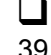

39

$82,8 \quad 8,3$

$n=462$

$n(k . A)=$. nein

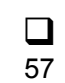

57
12,3 unent-

schieden

$\square$

41. Haben Sie im Rahmen Ihrer ärztlichen $n=470$

Tätigkeit in der Allgemeinmedizin gearbeitet?

$\begin{array}{lccc}\mathrm{n}=470 & & \text { ja } & \text { nein } \\ & & \square^{2} & \square \\ \mathrm{n}(\mathrm{k} . \mathrm{A} .)=4 & \mathrm{n} & 186 & 284 \\ & \% & 39,6 & 60,4\end{array}$


42. Ihre Abteilung

$$
\begin{array}{lrrl}
\mathrm{n}=467 & 120 & 25,7 & \square \text { Chirurgie } \\
\mathrm{n}(\mathrm{k} . \mathrm{A} .)=7 & 26 & 5,6 & \square \text { Dekan } \\
& 42 & 9,0 & \square \text { Gynäkologie } \\
& 126 & 27,0 & \square \text { Innere Medizin } \\
& 77 & 16,5 & \square \text { Pädiatrie } \\
& 76 & 16,3 & \square \text { Psychiat./ Psychosom. }
\end{array}
$$

\begin{tabular}{|c|c|c|c|}
\hline$n=468$ & & weiblich & männlich \\
\hline & $\mathrm{n}$ & 26 & $\overline{440}$ \\
\hline$n(k . A)=8$. & $\%$ & 5,6 & 94,4 \\
\hline
\end{tabular}

43. Ihr Geschlecht

44. Ihr Alter

$$
\begin{array}{llll}
\mathrm{n}=448 & & \mathrm{n} & \% \\
& \text { 30-39 Jahre } & 14 & 3,1 \% \\
\mathrm{n}(\mathrm{k} . \mathrm{A} .)=26 & \text { 40-49 Jahre } & 130 & 29,0 \%
\end{array}
$$

$\begin{array}{llr}\text { 30-39 Jahre } & 14 & 3,1 \% \\ \text { 40-49 Jahre } & 130 & 29,0 \% \\ \text { 50-59 Jahre } & 204 & 45,5 \% \\ \text { 60-69 Jahre } & 100 & 22,3 \%\end{array}$

Alter (min): 30 Jahre

Alter (max): 68 Jahre

Durchschnittsalter: 53,00 Jahre Median: 53 Jahre

45. Bundesland

$\begin{array}{lccl}\mathrm{n}=450 & \mathrm{n} & & \\ \mathrm{n}(\mathrm{k} . \mathrm{A} .)=24 & 14,2 & \square \text { Baden-Württemberg } \\ & 49 & 10,9 & \square \text { Bayern } \\ 34 & 7,6 & \square \text { Berlin } \\ 14 & 3,1 & \square \text { Hamburg } \\ 40 & 8,9 & \square \text { Hessen } \\ 22 & 4,9 & \square \text { Meck.-Vorpommern } \\ 33 & 7,3 & \square \text { Niedersachsen } \\ 99 & 22,0 & \square \text { NRW } \\ 8 & 1,8 & \square \text { Rheinland-Pfalz } \\ 9 & 2,0 & \square \text { Saarland } \\ 20 & 4,4 & \square \text { Sachsen } \\ 27 & 6,0 & \square \text { Sachsen-Anhalt } \\ 19 & 4,2 & \square \text { Schleswig-Holstein } \\ 12 & 2,7 & \square \text { Thüringen }\end{array}$




\subsection{Vollständige Auswertung: Bewertung primärmedizinischer Versorgung und Funktionen; nach Klinikzugehörigkeit}

Positive Aussage* $^{*} \quad$ Zustimmung

(\%)

\begin{tabular}{|c|c|c|c|c|c|c|}
\hline & Chirurgie & $\begin{array}{c}\text { Gynäko- } \\
\text { logie }\end{array}$ & $\begin{array}{l}\text { Innere } \\
\text { Medizin }\end{array}$ & Pädiatrie & $\begin{array}{l}\text { Psychiatrie } \\
\text { u. Psycho- } \\
\text { somatik }\end{array}$ & Dekan \\
\hline & $(n=120)$ & $(n=42)$ & $(n=126)$ & $(n=77)$ & $(n=76)$ & $(n=26)$ \\
\hline $\begin{array}{l}\text { Allgemeinärzte arbeiten } \\
\text { mit einer größeren } \\
\text { therapeutischen } \\
\text { Unsicherheit. }\end{array}$ & $75 \%$ & $71,4 \%$ & $72,8 \%$ & $68,8 \%$ & $70,3 \%$ & $57,7 \%$ \\
\hline $\begin{array}{l}\text { Allgemeinärzte stellen } \\
\text { die primärärztliche } \\
\text { Versorgung sicher. }\end{array}$ & $62,5 \%$ & $56,1 \%$ & $61,1 \%$ & $57,9 \%$ & $81,3 \%$ & $65,4 \%$ \\
\hline $\begin{array}{l}\text { Allgemeinärzte haben } \\
\text { eine wichtige Aufgabe } \\
\text { im Gesundheitssystem. }\end{array}$ & $83,2 \%$ & $92,9 \%$ & $85,7 \%$ & $86,8 \%$ & $91,9 \%$ & $80,8 \%$ \\
\hline $\begin{array}{l}\text { Allgemeinärzte sind } \\
\text { besonders befähigt, } \\
\text { chronisch Kranke zu } \\
\text { betreuen. }\end{array}$ & $63,6 \%$ & $57,1 \%$ & $36,5 \%$ & $32,5 \%$ & $62,7 \%$ & $65,4 \%$ \\
\hline $\begin{array}{l}\text { Allgemeinärzte sind } \\
\text { stärker durch } \\
\text { Patientenwünsche } \\
\text { beeinflußt. }\end{array}$ & $68,6 \%$ & $47,6 \%$ & $71,4 \%$ & $68 \%$ & $68 \%$ & $57,7 \%$ \\
\hline $\begin{array}{l}\text { Allgemeinmedizinische } \\
\text { Entscheidungsfindung } \\
\text { ist nicht unwissen- } \\
\text { schaftlich. }\end{array}$ & $26,9 \%$ & $29,3 \%$ & $23,8 \%$ & $20,8 \%$ & $29,7 \%$ & $30,7 \%$ \\
\hline $\begin{array}{l}\text { Allgemeinärzte sind } \\
\text { keine „Schmalspur- } \\
\text { mediziner“. }\end{array}$ & $89,1 \%$ & $90,2 \%$ & $72,6 \%$ & $76,3 \%$ & $74,3 \%$ & $80,8 \%$ \\
\hline
\end{tabular}

* Für die Definition positive Aussage gelten die in Kapitel 4.3 angegebenen Kriterien. 


\subsection{Vollständige Auswertung: Einstellung zur universitären Allgemeinmedizin; nach Klinikzugehörigkeit}

Positive Aussage*
Zustimmung

(\%)

\begin{tabular}{|c|c|c|c|c|c|c|}
\hline & $\begin{array}{l}\text { Chirurgie } \\
(n=120)\end{array}$ & $\begin{array}{l}\text { Gynäko- } \\
\text { logie } \\
(n=42)\end{array}$ & $\begin{array}{l}\text { Innere } \\
\text { Medizin } \\
(n=126)\end{array}$ & $\begin{array}{l}\text { Pädiatrie } \\
\text { (n=77) }\end{array}$ & $\begin{array}{l}\text { Psychiatrie } \\
\text { u. Psycho- } \\
\text { somatik } \\
(n=76)\end{array}$ & $\begin{array}{l}\text { Dekan } \\
(n=26)\end{array}$ \\
\hline $\begin{array}{l}\text { Die Bedeutung } \\
\text { allgemeinmedizinischer } \\
\text { Forschung ist der } \\
\text { anderer Fächer } \\
\text { vergleichbar. }\end{array}$ & $41,9 \%$ & $53,7 \%$ & $42,3 \%$ & $44,2 \%$ & $65,3 \%$ & $60 \%$ \\
\hline $\begin{array}{l}\text { Die Qualität } \\
\text { allgemeinmedizinischer } \\
\text { Forschung ist der } \\
\text { anderer Fächer } \\
\text { vergleichbar. }\end{array}$ & $12,5 \%$ & $19,1 \%$ & $13,6 \%$ & $10,7 \%$ & $17,6 \%$ & $20 \%$ \\
\hline $\begin{array}{l}\text { Sozialwissenschaftliche } \\
\text { und naturwissen- } \\
\text { schaftliche Methoden } \\
\text { sollten im Studium } \\
\text { gleichberechtigt sein. }\end{array}$ & $46,2 \%$ & $52,4 \%$ & $36,5 \%$ & $61,3 \%$ & $75,7 \%$ & $38,5 \%$ \\
\hline $\begin{array}{l}\text { Allgemeinärzte sind an } \\
\text { Forschung interessiert. }\end{array}$ & $28,2 \%$ & $21,3 \%$ & $26,6 \%$ & $17,1 \%$ & $15,1 \%$ & $46,2 \%$ \\
\hline $\begin{array}{l}\text { Allgemeinmedizinische } \\
\text { Forschungsergebnisse } \\
\text { sind auch für andere } \\
\text { Fächer relevant. }\end{array}$ & $67 \%$ & $66,7 \%$ & $62,6 \%$ & $70,7 \%$ & $79,7 \%$ & $68 \%$ \\
\hline $\begin{array}{l}\text { Die Bedeutung } \\
\text { allgemeinmedizinischer } \\
\text { Lehre ist der anderer } \\
\text { Fächer vergleichbar. }\end{array}$ & $69,7 \%$ & $73,2 \%$ & $76,9 \%$ & $80 \%$ & $81,1 \%$ & $88 \%$ \\
\hline $\begin{array}{l}\text { Die Qualität allgemein- } \\
\text { medizinischer Lehre ist } \\
\text { der anderer Fächer } \\
\text { vergleichbar. }\end{array}$ & $28,6 \%$ & $31 \%$ & $27,2 \%$ & $22,4 \%$ & $29,7 \%$ & $65,4 \%$ \\
\hline $\begin{array}{l}\text { Allgemeinmedizinische } \\
\text { Lehre ist methodisch } \\
\text { eigenständig. }\end{array}$ & $65,3 \%$ & $51,2 \%$ & $56,9 \%$ & $61,1 \%$ & $58,3 \%$ & $65,4 \%$ \\
\hline $\begin{array}{l}\text { Allgemeinmedizinische } \\
\text { Lehre ist inhaltlich } \\
\text { eigenständig. }\end{array}$ & $52,1 \%$ & $61,9 \%$ & $49,6 \%$ & $63,2 \%$ & $78,4 \%$ & $61,5 \%$ \\
\hline
\end{tabular}

* Für die Definition positive Aussage gelten die in Kapitel 4.3 angegebenen Kriterien. 


\section{Literaturverzeichnis}

Abholz HH: Forschungsbedarf in der primärärztlichen Versorgung; in: Primärärztliche Patientenbetreuung. Lehre-Forschung-Praxis; hrsg. v. Helmich P u.a.; Schattauer Verlag, Stuttgart 1997, 258-273

Abholz $\mathrm{HH}$ : Entscheidungsfindung in der Allgemeinmedizin.; in: Allgemeinmedizin; hrsg. v. Kochen MM; 2. Auflage; Hippokrates Verlag, Stuttgart 1998, 65-79

Abholz HH (1999): Argumente für ein Primärarztsystem. Z Allgemeinmed $\underline{75}$, 732-735

Abholz HH (2000a): Was ist eigentlich Allgemeinmedizin? Eine brisante Frage! Z Allgemeinmed $\underline{76}, 107$

Abholz HH (2000b): Allgemeinmedizin - gibt es ein einheitliches Fach, was wir in Lehre und Weiterbildung vermitteln? Z Allgemeinmed $\underline{76}$, 285-286

Abholz HH (2000c): Nachdenkliches über hausärztliche Leitlinien - gewonnen aus deren Entwicklung. Z Allgemeinmed $\underline{76}, 150-154$

Abrahamson $\mathrm{JH}$ : Making sense of data: a self-instruction manual on the interpretation of epidemiological data. Oxford University Press, Oxford 1988

Allen J, Wilson A, Fraser R, Pereira Grey D (1993): The academic base for general practice: the case for change. Br Med J $\underline{307}, 719-722$

Amon T, Wiesemann A, Klimm HD, Koser R, Schneider G, Sonntag HG (1997): Das Heidelberger Hospitationsprogramm. Z Allgemeinmed $\underline{73}, 1171-1176$

Babbie E: The practice of social research. 7. Auflage; Wadsworth Publishing Company, Belmont 1995

Bärlocher F: Biostatistik. Thieme Verlag, Stuttgart 1999

Becker A, Kochen MM (2001): Möglichkeiten und Grenzen der EBM. Z Allgemeinmed $\underline{77}$, 296-299

Benninghaus $\mathrm{H}$ : Einführung in die sozialwissenschaftliche Datenanalyse. 5. Auflage; R. Oldenbourg Verlag, München 1998

Bentham J, Burke J, Clarke J, Svoboda C, Vallance G, Yeow M (1999): Students conducting consultations in general practice and the acceptability to patients. Med Educ $\underline{33}, 686-687$

Beyer M, Gerlach FM, Rohde-Kampmann R (2000): DEGAM-Leitlinien für die hausärztliche Praxis - wie ist der Stand der Dinge? Z Allgemeinmed $\underline{76}$, 358-363

Blake RL (1999): Preface. Acad Med $\underline{74}$, Supplement, svii- sviii 
Bland CJ, Meurer LN, Maldonado G (1995): Determinants of primary care specialty choice: A non-statistical meta-analysis of the literature. Acad Med $\underline{70}$, 620-641

Block SD, Clark-Chiarelli N, Peters AS, Singer JD (1996): Academia`s chilly climate for primary care. JAMA $\underline{276}, 677-682$

Block SD, Clark-Chiarelli N, Singer JD (1998): Mixed messages about primary care in the culture of U.S. medical schools. Acad Med $\underline{73}, 1087-1094$

Bödecker A-W (2000): Barfuß oder mit voller Montur - womit die Lehrer der Allgemeinmedizin in ihren Praxen ausgestattet sind. Z Allgemeinmed $\underline{76}$, 287-289

Bortz J: Lehrbuch der empirischen Forschung für Sozialwissenschaftler. Springer Verlag, Berlin 1984

Bortz J, Döring N: Forschungsmethoden und Evaluation. 2. Auflage; Springer Verlag, Berlin 1995

Braun RN: Wissenschaftliches Arbeiten in der Allgemeinmedizin. Springer Verlag, Berlin 1988

Brinkley B (2001): Pourquoi une unité d'enseignement de médecine générale à Genève? Prim Care 1, 19-20

Brooks WB, Orgren R, Wallace AG (1999): Institutional change: embracing the initiative to train more generalists. Acad Med $\underline{74}$, Supplement, S3-S8

Brüning $\mathrm{H}$ : Allgemeinmedizinische Forschung in der Bundesrepublik Deutschland. Med. Diss. Marburg 1987

Brune M, Werle M, Hippler HJ (1991): Probleme bei der Befragung älterer Menschen. Methodische Erfahrungen aus einer schriftlichen Befragung zu Tätigkeitsformen im Ruhestand. ZUMA-Nachrichten $\underline{28}$, 73-91

Bühler G: Medizinstudium und Studienreform in der SBZ und der DDR (1945-1990). Med. Diss. Leipzig 1998

Bundesärztekammer: Approbationsordnung für Ärzte. Deutscher Ärzte-Verlag, Köln 1994

Bundesärztetag: Die Zukunft der Medizinischen Hochschulen. Köln 1996

(http://www.baek.de/30/Aerztetag/90 Beschluss/BeDAET99.pdf; accessed Januar 2002)

Bundesärztetag: Musterweiterbildungsordnung Allgemeinmedizin. Eisenach 1997

(http://www.baek.de/30/Aerztetag/90 Beschluss/BeDAET100.pdf; accessed Januar 2002) 
$\mathrm{BMBF}=$ Bundesministerium für Bildung und Forschung: Förderrichtlinien zur Förderung der „Forschung in der Allgemeinmedizin“ in den medizinischen Fakultäten und Hochschulen. Bonn 1999

Bundesministerium für Gesundheit: Reform der ärztlichen Ausbildung. Bonn 1997 (http://www.bmgesundheit.de/themen/berufe/voha/berufe/reform.htm;accessed Januar 2002) Bundesministerium für Gesundheit: Ärztliche Ausbildung in der Bundesrepublik Deutschland. Berlin 2001 (http://www.bmgesundheit.de/themen/berufe/arzt/ubersich.htm; accessed Januar 2002) Bundesministerium für Gesundheit: Reform des Medizinstudiums macht Arztberuf wieder attraktiver. Pressemitteilung Nr.44 vom 26.April 2002. Berlin 2002 (http://www.bmgesundheit.de/presse/2002/2002/44.htm; accessed April 2002)

Carter YH, Hilton S (2000): Academic general practice: no time for ivory towers. Fam Pract $\underline{17}, 283-284$

Carter Y, Elvyn G: History and structure of research in primary care; in: Oxford textbook of primary medical care; hrsg. v. Jones R u.a.; Oxford University Press, Oxford 2002 (in press)

Clade H (1997a): Reform des Medizinstudiums. „Der Fakultätentag blockiert.“ Dtsch Ärztebl $\underline{94}$, A 498-499

Clade H (1997b): Reform des Medizinstudiums. Gesucht: Tragfähiger Entwurf. Dtsch Ärztebl 94, A 1848-1849

Clade H (2001): Reform des Medizinstudiums. Auf der Zielgeraden. Dtsch Ärztebl $\underline{98}, \mathrm{C}$ 1620-1624

Comberg HU (1994): Was ist Allgemeinmedizin? Z Ärztl Fortb $\underline{88}, 639-642$

Coulter A (1996): Why should health care services be primary care-led? J Health Serv Res Policy $\underline{1}, 122-124$

Curtis P, Smith AC (1997): Medical school culture and generalist education: perceptions of a medical school faculty. Fam Med $\underline{29}$, 353-358

Dekanat der Universität Regensburg: mündliche Mitteilung 30.6.1999

DEGAM = Deutsche Gesellschaft für Allgemeinmedizin (2002a): Musterweiterbildungsordnung - Allgemeinmedizin. (http://www.degam.de/weiterbildung/wbo.htm, accessed Januar 2002) 
DEGAM = Deutsche Gesellschaft für Allgemeinmedizin (2002b): Wie plane ich meine Weiterbildung zum Allgemeinarzt? (http://www.degam.de/weiterbildung/plan.htm, accessed Januar 2002)

Dillman DA: Mail and telephone surveys: The total design method. John Wiley \& Sons, New York 1978

Donner-Banzhoff N (2000): Puzzle 2000: Die Novellierung der Weiterbildungsordnung. Z Allgemeinmed $\underline{76}, 199-202$

Donner-Banzhoff N, Abholz HH (2000): Vorschlag der DEGAM zur Weiterbildung Allgemeinmedizin: die Verbundlösung. Z Allgemeinmed $\underline{76}$, 290-292

Edwars AL: The social desirability variable in personality assessment and research, Holt Press, New York, 1957

Ely JW, Dawson JD, Mehr DR, Burns TL (1996): Understanding logistic regression analysis through example. Fam Med 28, 134-140

Engel GL (1982): The biopsychosocial model and medical education. N Engl J Med $\underline{306}$, 802-805

Engström S, Foldevi M, Borgquist L (2001): Is general practice effective? A systematic literature review. Scand J Prim Health Care 19, 131-144

von Ferber C (1989): Die akademische Etablierung der Allgemeinmedizin. Z Allgemeinmed $\underline{65}, 319-323$

Fischer GC, Gerlach FM, Pangritz J, Pullwitt DH, Rossa B (1993): Allgemeinmedizin an deutschen Hochschulen. Dtsch Ärztebl 90, C 1071-1074

Freeman AC, Sweeney K (2001): Why general practitioners do not implement evidence: qualitative study. Br Med J $\underline{323}, 1-5$

Friedrichs J: Methoden empirischer Sozialforschung. 14. Auflage; Westdeutscher Verlag, Opladen 1990

Geyer S, Siegrist J: Sozialwissenschaftliche Verfahren in den Gesundheitswissenschaften; in: Handbuch der Gesundheitswissenschaften; hrsg. v. Hurrelmann K, Laaser U; JuventaVerlag, Weinheim 1998, 259-279

Gill P, Dowell AC, Neal RD, Smith N, Heywood P, Wilson AE (1996): Evidence based general practice: a retrospective study of interventions in one training practice. $\mathrm{Br}$ Med J $\underline{312}$, 819-821

Goßmann HH (1992): Das Besondere am Allgemeinen. Dtsch Ärztebl 모, A1, 668-671 
Grayson MS, Newton DA, Klein M, Irons T (1999): Promoting institutional change to encourage primary care: Experiences at New York Medical College and East Carolina University School of Medicine. Acad Med $\underline{74}$, Supplement, S9-S15

Greenfield S, Rogers W, Mangotich M, Carney MF, Tarlov AR (1995): Outcomes of patiemts with hypertension and non-insulin dependent diabetes mellitus treated by different systems and specialties. Results from the medical outcomes study. JAMA $\underline{274}, 1436-1444$

Grupp R: Reformziele bei der Ausbildung der Ärzte; in: Primärärztliche Patientenbetreuung. Lehre-Forschung-Praxis; hrsg. v. Helmich P u.a.; Schattauer Verlag, Stuttgart 1997, 88-92

Günther E: Der Hausarzt in der DDR - was ist er und was wird aus ihm?; in: Das Gesundheitswesen der DDR. Aufbruch oder Einbruch? Denkanstösse für eine Neuordnung des Gesundheitswesens in einem deutschen Staat; hrsg. v. Thiele W; Asgard-Verlag, Sankt Augustin 1990, 138-142

Gulich M, Zeitler HP (1999): Allgemeinmedizin als Wahlfach im Praktischen Jahr? Z Allgemeinmed $\underline{75}, 762-762$

Habeck D (1992): Die Lehre der Allgemeinmedizin im deutschen Medizinstudium. Med Ausbild $\underline{9}, 81-90$

Härter G, Dieckhoff D, Faust G, Pillau H, Riese W (1990): Lehrstoffkatalog Allgemeinmedizin und Familienmedizin. Internationale Allgemeinmedizin und Hochschule. Beilage zur Z Allgemeinmed 24, I 1515-26

Hagedorn M (1989): Die Rechtsstellung des Lehrbeauftragten für Allgemeinmedizin. Z Allgemeinmed $\underline{65}$,

Harrold LR, Field TS, Gurwitz JH (1999): Knowledge, patterns of care, and outcomes of care for generalists and specialists. J Gen Intern Med 14, 499-511

Harych H: Zur Zukunft der Polikliniken und der ambulanten Versorgung in der DDR; in: Das Gesundheitswesen der DDR. Aufbruch oder Einbruch? Denkanstösse für eine Neuordnung des Gesundheitswesens in einem deutschen Staat; hrsg. v. Thiele W; Asgard-Verlag, Sankt Augustin 1990, 99-104

Heimpel $\mathrm{H}$ : Kriterien für Ausbildungsinhalte und Ausbildungsformen aus der Sicht eines Internisten; in: Primärärztliche Patientenbetreuung. Lehre-Forschung-Praxis; hrsg. v. Helmich P u.a.; Schattauer Verlag, Stuttgart 1997, 160-168 
Helmich P: Vom Allgemeinmediziner zum Hochschullehrer. Überlegungen zur akademischen Laufbahn allgemeinmedizinischer Ärztinnen und Ärzte; in: Primärärztliche Patientenbetreuung. Lehre-Forschung-Praxis; hrsg. v. Helmich P u.a.; Schattauer Verlag, Stuttgart 1997, 69-86

Hilton S, Carter YH (2000): Academic careers in general practice and primary care. Med Edu $\underline{34}, 910-915$

Himmel W (2000): Allgemeinmedizinischer Unterricht in der Praxis. Z Allgemeinmed $\underline{76}, 192$

Himmel W, Kochen MM (1997): Habilitation im Fachgebiet Allgemeinmedizin. Z Allgemeinmed $\underline{73}, 437-442$

Himmel W, Dieterich A, Kochen MM (2000): Will german patients accept their family physician as a gatekeeper? J Gen Intern Med $\underline{15}, 496-502$

Himmel W, Rogausch A, Kochen MM: Principles of patient management; in: Oxford textbook of primary medical care; hrsg. v. Jones R u.a.; Oxford University Press, Oxford 2002: im Druck

Hippler H-J: Schriftliche Umfragen bei repräsentativen Bevölkerungsstichproben oder: Wie erreicht man 78\%?; in: Herausforderungen der empirischen Sozialforschung; hrsg. v. Kaase M und Küchler M, ZUMA, Mannheim 1985, 71-74

Hippler H-J (1988): Methodische Aspekte schriftlicher Befragungen: Probleme und Forschungsperspektiven. Planung \& Analyse $\underline{6}, 244-248$

Howe A, Galen I (2001): Does community-based experience alter career preference? New evidence from a prospective longitudinal cohort study of undergraduate students. Med Educ 35, 391-397

Hummers-Pradier E, Kochen MM (2000): DEGAM-Leitlinie Nr.1 „Brennen beim Wasserlassen“. Z Allgemeinmed 76, 35-48 (http://www.degam.de/S5 leit1.html; accessed Januar 2002)

Hummers-Pradier E, Gerlach FM, Kochen MM (2000): Von der wissenschaftlichen Evidenz zur praxisgerechten Leitlinie: Die Entstehung der Leitlinie „Brennen beim Wasserlassen“. Z Allgemeinmed $\underline{76}, 94-97$

Hummers-Pradier E, Becker A, Maitra RT (2002): Wissenschaftlicher Nachwuchs in der Allgemeinmedizin - am Rande der Legalität? Z Allgemeinmed $\underline{78}, 32-36$

Hungeling G (1995): Allgemeinmedizin im Studium. Z Allgemeinmed 71, 1365-1372 Infratest, München: schriftliche Mitteilung 2001 
Jolly B, Rees L: Medical education in the millenium. Oxford University Press, Oxford 1998 Jones R (2000): Primary care research: ends and means. Fam Pract 17: 1-4

Jones S, Oswald N (1996): Attitudes of patients to medical students participation: general practice consultations on the Cambridge community-based clinical. Med Educ $\underline{30}$, 14-17

Joosten U: Die Entwicklung der Allgemeinmedizin als Wissenschaft seit 1969. Med.Diss. Bochum 1995

Kassenärztliche Bundesvereinigung: Ärztestatistik zum 31.12.2000. O. Ort, o. Jahr. (http://www.kbv.de/publikationen/190.htm ; accessed Januar 2002)

Klein S, Porst R: Mail Surveys. Ein Literaturbericht. ZUMA - Technischer Bericht 10/2000. ZUMA, Mannheim 2000

Klimm HD (1994): Einführung zum Themenheft „Allgemeinmedizin“. Z Ärztl Fortb $\underline{88}, 635-$ 637

Kochen MM: Forschung in der Allgemeinmedizin. In: Allgemeinmedizin; hrsg. v. Kochen MM; 2. Auflage; Hippokrates Verlag, Stuttgart 1998, 518-526

Kochen MM (1989): Allgemeinmedizin an die Universitäten! Z Allgemeinmed $\underline{65}, 3$

Kochen MM (1999): New impulses for general practice in Germany. Eur J Gen Pract $\underline{5}, 10$

Kochen MM (2002): Vorschlag zu einer Kriterienliste "Qualifikation eines Hochschullehrers“. Z Allgemeinmed $\underline{78}$, (in Druck)

Kochen MM, Himmel W (2000): Academic careers in general practice: scientific requirements in Europe. Eur J Gen Pract $\underline{6}$, 62-65

Kochen MM, Fischer GC, Jork K, Kruse W, Pillau H (1990): Allgemeinmedizin als Hochschulfach. Z Allgemeinmed $\underline{66}, 588-594$

Kochen MM, Niebling W, Abholz HH (2000): Forschen oder beforscht werden? Z Allgemeinmed $\underline{76}, 347-348$

Kopetsch T (2002): Gehen dem deutschen Gesundheitswesen die Ärzte aus ?

Kassenärztliche Bundesvereinigung Archivnummer 1003736948

(http://www.kbv.de/publikationen/2690.htm; accessed Januar 2002)

Korzilius H (2001a): Ja zur Hausarzt-Philosophie. Dtsch Ärztebl 98, C 1669-1670

Korzilius H (2001b): Hausärztliche Versorgung. Vorschlag zur Güte. Dtsch Ärztebl $\underline{98}$, C 2369

Last JM (1963): The iceberg: completing the picture in general practice. Lancet 1963,2,28-31 
Levasseur G, Schweyer FX, Perriniaux J (2001): Research in general practice in France today. Eur J Gen Pract $\underline{7}, 62-67$

Van de Lisdonk EH (1989): Perceived and presented morbidity in general practice. Scand J Prim Health Care $\underline{7}, 73$

Mainous III AG, Hueston WJ, Xiaobu Y, Bazell C (2000): A comparison of family medicine research in research intense and less intense institutions. Arch Fam Med $\underline{9}, 1100-1104$

Marzi C, Abholz HH (1999): Hinweise für die Überlegenheit eines Primärarztsystems. Z Allgemeinmed $\underline{75}, 736-743$

Maronde H-U, Sander L (1989): Der Stellenwert der Allgemeinmedizin in der Beurteilung von Studenten der Grundstudieneinrichtung Medizin - Ergebnisse einer Befragung. Z Klin Med $\underline{44}, 2129-2131$

Maus J (2001a): Internisten machen gegen das Hausarztmodell mobil. Dtsch Ärztebl $\underline{98}$, C 1432

Maus J (2001b): Aufforderung zum Duell. Dtsch Ärztebl 98, C 1536

Maus J (2001c): Hausärztliche Versorgung: Optimistisch für die Zukunft. Dtsch Ärztebl $\underline{98}, \mathrm{C}$ 2094

Morrison J, Watt G (2001): Primary care research: educational and development needs. Med Educ $\underline{35}, 5$

Muche R, Bopp T, Gaus W (2000): Klinische Studien in der Praxis: Notwendigkeit, Grundlagen und Durchführbarkeit - eine Erhebung in 123 Arztpraxen. Z Allgemeinmed $\underline{76}$, 352-355 Murrhardter Kreis - Arbeitskreis Medizinerausbildung der Robert Bosch Stiftung: Das Arztbild der Zukunft. 3. Auflage; Bleicher Verlag, Gerlingen 1995

Niemann H, Wiezorek WD (1980a): Zur Geschichte der medizinischen Hochschulbildung in der Deutschen Demokratischen Republik. Teil I. Z Ärztl Fortbild $\underline{74}, 115-122$

Niemann H, Wiezorek WD (1980b): Zur Geschichte der medizinischen Hochschulbildung in der Deutschen Demokratischen Republik. Teil II. Z Ärztl Fortbild $\underline{74}, 178-181$

Nutting PA (1996): Practice-based research networks: building the infrastructure of primary care research. J Fam Pract 42, 199-203

Nutting PA, Beasley JW, Werner JJ (1999): Practice-based research networks answer primary care questions. JAMA $\underline{24}, 686-688$

Parle JV, Greenfield SM, Skelton J, Lester H, Hobbs FDR (1997): Acquisition of basic clinical skills in general practice setting. Med Educ $\underline{31}, 99-104$ 
Pauli HG (1996): Wird das Medizinstudium der Aufgabe des Arztes gerecht?. Schweiz Rundsch Med (Praxis) $\underline{85}$, 877-882

Perleth M (1998): Ärztliche Basiskompetenzen: Vernachlässigtes Ausbildungsziel im Medizinstudium? Med Klin $\underline{93}$, 381-87

Pönicke W: Allgemeinmedizinische Tätigkeit - hausärztliche Betreuung; in: Das Gesundheitswesen der DDR. Aufbruch oder Einbruch? Denkanstösse für eine Neuordnung des Gesundheitswesens in einem deutschen Staat; hrsg. v. Thiele W; Asgard-Verlag, Sankt Augustin 1990, 143-145

Porst R: Wie man die Rücklaufquote bei postalischen Befragungen erhöht. ZUMA How-toReihe Nr. 09, ZUMA, Mannheim 2001

Porst R, Ranft S, Ruoff B: Strategien und Maßnahmen zur Erhöhung der Ausschöpfquoten bei sozialwissenschaftlichen Umfragen. Ein Literaturbericht. ZUMA-Arbeitsbericht Nr. 98/07, ZUMA, Mannheim 1998

Pugno P, Schmittling GT, Mc Pherson DS, Kahn NB (2000): Entry of US medical school graduates into family practice residencies: 1999-2000 and a 3-year summary. Fam Med $\underline{32}$, 534-542

Pugno P, Schmittling GT, Mc Pherson DS, Kahn NB (2001): Entry of US medical school graduates into family practice residencies: 2000-2001 and a 3-year summary. Fam Med $\underline{33}$, 585-593

Richter EA (2001a): Mehr Praxis, weniger Multiple Choice. Dtsch Ärzteblatt 98, C 1622-23

Richter EA (2001b): Facharzt/Hausarzt. Studie sorgt für Wirbel. Dtsch Ärztebl 98, C 2144

Rossi PH, Wright JD, Anderson AB: Handbook of survey research. Academic Press, San Diego 1983

Roth E: Sozialwissenschaftliche Methoden. 3. Auflage; R. Oldenbourg Verlag, München 1993

Sahlmann P (2000): Untersuchung zum Verhalten von Medizinstudenten in einer Praxis für Allgemeinmedizin im Rahmen eines Praktischen Tages. Z Allgemeinmed $\underline{76}$, 196-198

SAS Institute Inc.: SAS/STAT. User's guide. Version 8. SAS Institute Inc., Cary/North Carolina 2000

Schafer S, Shore W, French L, Tovar J, Hughes S, Hearst N (2000): Rejecting family practice: why medical students switch to other specialties. Fam Med 32(5), 320-325 
Scheffner D: Primärärztliche Kompetenz als Ausbildungsziel im Reformstudiengang Medizin in Berlin; in: primärärztliche Patientenbetreuung. Lehre-Forschung-Praxis; hrsg. v. Helmich P u.a.; Schattauer Verlag, Stuttgart 1997, 93-102

Schnell R, Hill PB; Esser E: Methoden der empirischen Sozialforschung. 6. Auflage, R. Oldenbourg Verlag, München 1999

Schrömbgens HH (1990): Hausarzt und Hochschullehrer. Z Allgemeinmed $\underline{66}, 595-599$

Schwenk TL (1998): Strangers in a strange land: primary care physicians in academic medical centers. J Gen Intern Med 13, 216-217

Senf JH, Campos-Outcalt D, Watkins AJ, Bastacky S, Killian C (1997): A systematic analysis of how medical school characteristics relate to graduates' choices of primary care specialties. Acad Med $\underline{72}, 524-533$

Siebolds M, Gesenhues S, Hermann M, Quellmann T, Webler WD, Berndt P (2000): Qualitätssicherung der allgemeinmedizinischen Lehre im Medizinstudium. Z Allgemeinmed $\underline{76}, 618-622$

Simons RJ, Imboden E, Martel JK (1995): Patient attitudes toward medical student participation in a general internal medicine clinic. J Gen Intern Med $\underline{10}$, 251-254

Sohn W: Die Lehre der Allgemeinmedizin. Rahmenbedingungen und Umsetzung im Düsseldorfer Lehrmodell; in: Primärärztliche Patientenbetreuung. Lehre-Forschung-Praxis; hrsg. v. Helmich P u.a.; Schattauer Verlag, Stuttgart 1997, 142-159

Starfield B (1991): Primary care and health, a cross-national comparison. JAMA $\underline{266}$, 22682271

Starfield B (1994): Is primary care essential? Lancet $\underline{334}, 1129-1133$

Starfield B (1997): The future of primary care in a managed care era. Int J Health Serv $\underline{27}$, 687-689

Steinkohl M (1996): Die Rolle der Allgemeinmedizin innerhalb des Gesundheitsversorgungssystems. Z Ärztl Fortbild $\underline{90}$, 153-157

Stokes ME, Davis CS, Koch GG: Categorical data analysis using the SAS system. SAS Institute Inc., Cary/ North Carolina 1995, 165-214

Stunder WA (2000): Akzeptanz von Weiterbildungsassistenten und Studenten in der Hausarztpraxis. Z Allgemeinmed $\underline{76}$, 193-195

Sturm E: Renaissance des Hausarztes. Springer Verlag, Berlin 1983 
Sullivan FM, Morrison JM (1997): What can universities do to reverse the decline in the numbers of doctors entering general practice? Med Educ $\underline{31}, 235-236$

Svab I, Kvetoslav S, Crebolder H (2001): General practice teaching and basic medical education in Europe. Eur J Gen Pract $\underline{7}, 112-114$

Thoma M, Zimmermann M (1996): Zum Einfluß der Befragungstechnik auf den Rücklauf bei schriftlichen Umfragen. Experimentelle Befunde zur „Total-Design-Method“. ZUMANachrichten $\underline{39}, 141-157$

University of Minnesota, Residency Programs. (http://www.med.umn.edu/fp/resy/rprur.htm; accessed Januar 2002)

Vereinigung der Hochschullehrer und Lehrbeauftragten für Allgemeinmedizin e.V., Aachen: 53. Semesterbericht über die Unterrichtsveranstaltungen Allgemeinmedizin im Wintersemester 2000/2001 und im Sommersemester 2001. Aachen 2001

Wagner EH, Austin BT, Von Korff M (1996): Organizing care for patients with chronic illness. Milbank Q $\underline{74}, 511-544$

van Weel C (1999): International research and the discipline of family medicine. Eur J Gen Pract $\underline{5}, 110-115$

van Weel C, Knottnerus JA (1999): Evidence-based interventions and comprehensive treatment. Lancet $\underline{353}, 916-918$

van Weel C, Smith H, Beasley JW (2000): Family practice research networks. Experiences from 3 countries. J Fam Pract $\underline{49}$, 938-943

$\mathrm{WHO}=$ World Health Organisation: Declaration of Alma-Ata, International Conference on Primary Health Care, Alma-Ata, USSR 6-12 September 1978: Website (www.who.int/hpr/docs/almaata.html; accessed Januar 2002)

Wilm S: Die Sozialisation zum Allgemeinarzt in Deutschland. In: Soziale Medizin, Jahrbuch für Kritische Medizin 26, Argument-Verlag, Hamburg 1997, 121-134

Wirsching $\mathrm{M}$, Fritzsche $\mathrm{K}$ : Was ist psychosomatische Grundversorgung? Ein obligates Thema der Weiter- und Fortbildung in allen Fachgebieten und die Fähigkeit zur Kooperation im Gesundheitssystem; in: Primärärztliche Patientenbetreuung. Lehre-Forschung-Praxis; hrsg. v. Helmich P u.a.; Schattauer Verlag, Stuttgart 1997, 178-186

Wissenschaftsrat: Leitlinien zur Reform des Medizinstudiums. O.Verl., Köln 1992.

Wissenschaftsrat: Stellungnahme zu den Perspektiven des Faches Allgemeinmedizin an den Hochschulen. O. Verl., Berlin 1999 
Zinn WM, Block SD, Clark-Chiarelli N (1998): Enthusiasm for primary care. J Gen Intern Med $13,186-194$

Zinn WM, Sullivan AM, Zotov N, Peters AS, Connelly MT, Singer JD (2001): The effect of medical education on primary care orientation: results of two national surveys of students" and residents' perspectives. Acad Med $\underline{76}, 355-365$ 


\section{Danksagung}

Ich danke Herrn Prof. Dr. med. Michael M. Kochen, MPH, FRCGP und meinem Doktorvater, Priv. Doz. Dr. disc. pol. Wolfgang Himmel, für die freundliche Überlassung des Themas der Dissertation.

Mein ganz besonderer Dank gilt Herrn Dr. Himmel für die ausgezeichnete Betreuung und die vielen wichtigen Anregungen. 


\section{Lebenslauf}

Ich wurde am 15.5.1971 in Gießen als erste Tochter von Siegfried und Sigrid Josupeit, geb. Haas, geboren. Von 1977-1981 besuchte ich die Schloßberg-Schule in Bensheim-Auerbach und anschließend das Alte Kurfürstliche Gymnasium in Bensheim, wo ich 1990 mein Abitur ablegte.

Während eines freiwilligen sozialen Jahres 1990/91 arbeitete ich in der offenen Altenhilfe der Gethsemane-Gemeinde Frankfurt/Main (Träger: Diakonisches Werk in Hessen und Nassau). Von 1991 bis zum Sommersemester 1994 studierte ich an der Johann Wolfgang Goethe-Universität Mittlere und Neuere Geschichte, Französisch und Evangelische Theologie (Magisterstudiengang).

1994 begann ich mein Medizinstudium an der Georg-August Universität Göttingen, das ich im Herbst 2000 mit dem dritten Staatsexamen abschloss. Im Rahmen meines Praktischen Jahres absolvierte ich ein Tertial in Innerer Medizin am Centre Hospitalier Universitaire in Montpellier/Frankreich und jeweils zwei Monate des Wahlfaches Pädiatrie am Centre Hospitalier Universitaire Montpellier/Frankreich und am Hospital for Sick Children, Toronto/Kanada.

Als Ärztin im Praktikum arbeitete ich von März 2001 bis Juni 2002 in der Inneren Abteilung des Marienkrankenhauses Trier-Ehrang und wechselte zum Juli 2002 in die Radiologische Abteilung des Krankenhauses der Barmherzigen Brüder Trier. 